ALEA, Lat. Am. J. Probab. Math. Stat. 15, 21-48 (2018)

DOI: $10.30757 /$ ALEA.v15-02

\title{
On scaling limits of multitype Galton-Watson trees with possibly infinite variance
}

\author{
Gabriel Hernán Berzunza Ojeda \\ Institut für Mathematik, Universität Zürich \\ Winterthurerstrasse 190, CH-8057 \\ Zürich, Switzerland. \\ E-mail address: gabriel.berzunza@math.uzh.ch \\ URL: http://stochastik.math.uni-goettingen.de/ gberzunza/
}

\begin{abstract}
In this work, we study asymptotics of multitype Galton-Watson forests with finitely many types. We consider critical and irreducible offspring distributions such that they belong to the domain of attraction of a stable law, where the stability indices may differ. We show that after a proper rescaling, their corresponding height process converges to the continuous-time height process associated with a strictly stable spectrally positive Lévy process. This gives an analog of a result obtained by Miermont (2008) in the case of multitype Galton-Watson forests with finite covariance matrices of the offspring distribution. Our approach relies on a remarkable decomposition for multitype trees into monotype trees introduced in Miermont (2008).
\end{abstract}

\section{Introduction}

In the pioneer works Aldous (1991, 1993), Aldous introduced the continuum random tree as the limit of rescaled Galton-Watson (GW) trees conditioned on the total progeny for offspring distributions having finite variance. Specifically, he proved that their properly rescaled contour functions converge in distribution in the functional sense to the normalized Brownian excursion, which codes the continuum random tree as the contour function does for discrete trees. This work has motivated the study of the convergence of other rescaled paths obtained from GW trees, and more generally GW forests, possibly with infinite variance, such as the Lukasiewicz path and the height process. Duquesne and Le Gall (2002) obtained in full generality an unconditional version of Aldous' result for trees and forests. More precisely, they showed that the concatenation of rescaled height processes (or

Received by the editors June 21th, 2016; accepted October 25th, 2017.

2010 Mathematics Subject Classification. 60J80, 60F17.

Key words and phrases. Multitype Galton-Watson tree; Height process; Scaling limit; Continuum random tree.

Research supported by the Swiss National Science Foundation 200021_144325/1. 
rescaled contour functions) converges in distribution to the so-called continuoustime height process associated to a spectrally positive Lévy process. In particular, when the offspring distribution belongs to the domain of attraction of a stable law of index $\alpha \in(1,2]$, Duquesne (2003) showed that the height processes of GW trees conditioned on having $n$ vertices converge in distribution to the normalized excursion of the continuous-time height process associated with a strictly stable spectrally positive Lévy process of index $\alpha$.

The present work has been motivated by the following result of Miermont (2008), which extends the previous ones on monotype GW trees to multitype GW trees and forests. Recall that multitype GW trees are a generalization of usual GW trees (or forests) that describe the genealogy of a population where individuals are differentiated by types that determine their offspring distribution. More precisely, Miermont establishes an unconditional version for the convergence of the rescaled height process of critical multitype GW forests with finitely many types to the reflected Brownian motion, under the hypotheses that the offspring distribution is irreducible and has finite covariance matrix. Moreover, under an additional exponential moment assumption, similar results are proved for GW trees conditioned on the number individuals of a given type, the limit of the height process is given by the normalized Brownian excursion. More recently, de Raphélis (2017) has extended the unconditional result in Miermont (2008) for multitype GW forests with infinitely many types, under similar assumptions. Informally speaking, these results claim that multitype GW forests behave asymptotically in a similar way as the monotype ones, at least in the finite variance case. Therefore, this suggests that we should expect an analogous behavior for multitype GW forests that satisfy weaker hypotheses.

Our main goal is to show an unconditional result for critical multitype GW forests with finitely many types whose offspring distribution is still irreducible, but may have infinite variance. Specifically, we are interested in establishing scaling limits for their associated height processes, when the offspring distributions belong to the domain of attraction of a stable law where the stability indices may differ. This will lead us to modify and extend the results of Miermont (2008).

In the rest of the introduction, we will describe our setting more precisely and give the exact definition of multitype GW trees and forests. We then provide the main assumptions on the offspring distribution in Section 1.2. This will enable us to state our main results in Section 1.4.

1.1. Multitype plane trees and forests. We recall the standard formalism for family trees. Let $U$ be the set of all labels:

$$
U=\bigcup_{n=0}^{\infty} \mathbb{N}^{n},
$$

where $\mathbb{N}=\{1,2, \ldots\}$ and with the convention $\mathbb{N}^{0}=\{\varnothing\}$. An element of $U$ is a sequence $u=u_{1} \cdots u_{j}$ of positive integers, and we call $|u|=j$ the length of $u$ (with the convention $|\varnothing|=0$ ). If $u=u_{1} \cdots u_{j}$ and $v=v_{1} \cdots v_{k}$ belong to $U$, we write $u v=u_{1} \cdots u_{j} v_{1} \cdots v_{k}$ for the concatenation of $u$ and $v$. In particular, note that $u \varnothing=\varnothing u=u$. For $u \in U$ and $A \subseteq U$, we let $u A=\{u v: v \in A\}$, and we say that $u$ is a prefix (or ancestor) of $v$ if $v \in u U$, in which case we write $u \vdash v$. Recall that 
the set $U$ comes with a natural lexicographical order $\prec$, such that $u \prec v$ if and only if either $u \vdash v$, or $u=w u^{\prime}, v=w v^{\prime}$ with nonempty words $u^{\prime}, v^{\prime}$ such that $u_{1}^{\prime}<v_{1}^{\prime}$.

A rooted planar tree $\mathbf{t}$ is a finite subset of $U$ which satisfies the following conditions:

I. $\varnothing \in \mathbf{t}$, we called it the root of $\mathbf{t}$.

II. For $u \in U$ and $i \in \mathbb{N}$, if $u i \in \mathbf{t}$ then $u \in \mathbf{t}$, and $u j \in \mathbf{t}$ for every $1 \leq j \leq i$.

We let $\mathbb{T}$ be the set of all rooted planar trees. We call vertices (or individuals) the elements of a tree $\mathbf{t} \in \mathbb{T}$, the length $|u|$ is called the height of $u \in \mathbf{t}$. We write $c_{\mathbf{t}}(u)=\max \left\{i \in \mathbb{Z}_{+}: u i \in \mathbf{t}\right\}$ for the number of children of $u$. The vertices of $\mathbf{t}$ with no children are called leaves. For $\mathbf{t}$ a planar tree and $u \in \mathbf{t}$, we let $\mathbf{t}_{u}=\{v \in U: u v \in \mathbf{t}\}$ be the subtree of $\mathbf{t}$ rooted at $u$, which is itself a tree. The remaining part $[\mathbf{t}]_{u}=\{u\} \cup\left(\mathbf{t} \backslash u \mathbf{t}_{u}\right)$ is called the subtree of $\mathbf{t}$ pruned at $u$. The lexicographical order $\prec$ will be called the depth first order on $\mathbf{t}$.

In addition to trees, we are also interested in forests. A forest $\mathbf{f}$ is a nonempty subset of $U$ of the form

$$
\mathbf{f}=\bigcup_{k} k \mathbf{t}_{(k)}
$$

where $\left(\mathbf{t}_{(k)}\right)$ is a finite or infinite sequence of trees, which are called the components of $\mathbf{f}$. In words, a forest may be thought of as a rooted tree where the vertices at height one are the roots of the forest components. We let $\mathbb{F}$ be the set of rooted planar forests. For $\mathbf{f} \in \mathbb{F}$, we define the subtree $\mathbf{f}_{u}=\{v \in U: u v \in \mathbf{f}\} \in \mathbb{T}$ if $u \in \mathbf{f}$, and $\mathbf{f}_{u}=\emptyset$ otherwise. Also, let $[\mathbf{f}]_{u}=\{u\} \cup\left(\mathbf{f} \backslash u \mathbf{f}_{u}\right) \in \mathbb{F}$. With this notation, we observe that the tree components of $\mathbf{f}$ are $\mathbf{f}_{1}, \mathbf{f}_{2}, \ldots$ We let $c_{\mathbf{f}}(u)$ be the number of children of $u \in \mathbf{f}$. In particular, $c_{\mathbf{f}}(\varnothing) \in \mathbb{N} \cup\{\infty\}$ is the number of components of f. We call $|u|-1$ the height of $u \in \mathbf{f}$. Notice that that notion of height differs from the convention on trees because we want the roots of the forest components to be at height 0 .

Let $d \in \mathbb{N}$, we call $[d]=\{1, \ldots, d\}$ the set of types. A $d$-type planar tree, or simply a multitype tree is a pair $\left(\mathbf{t}, e_{\mathbf{t}}\right)$, where $\mathbf{t} \in \mathbb{T}$ and $e_{\mathbf{t}}: \mathbf{t} \rightarrow[d]$ is a function such that $e_{\mathbf{t}}(u)$ corresponds to the type of a vertex $u \in \mathbf{t}$. We let $\mathbb{T}^{(d)}$ be the set of $d$-type rooted planar trees. For $i \in[d]$, we write $c_{\mathbf{t}}^{(i)}(u)=\#\{j \in$ $\mathbb{Z}_{+}: u j \in \mathbf{t}$ and $\left.e_{\mathbf{t}}(u j)=i\right\}$ for the number of offspring of type $i$ of $u \in \mathbf{t}$. Then, $c_{\mathbf{t}}(u)=\sum_{i \in[d]} c_{\mathbf{t}}^{(i)}(u)$ is the total number of children of $u \in \mathbf{t}$. Analogous definitions hold for $d$-type rooted planar forests $\left(\mathbf{f}, e_{\mathbf{f}}\right)$, whose set will be denoted by $\mathbb{F}^{(d)}$. For sake of simplicity, we shall frequently denote the type functions $e_{\mathbf{t}}, e_{\mathbf{f}}$ by $e$ when it is free of ambiguity, and will even denote elements of $\mathbb{T}^{(d)}, \mathbb{F}^{(d)}$ by $\mathbf{t}$ or $\mathbf{f}$, without mentioning $e$. Moreover, it will be understood then that $\mathbf{t}_{u}, \mathbf{f}_{u},[\mathbf{t}]_{u},[\mathbf{f}]_{u}$ are marked with the appropriate function.

Finally, for $\mathbf{t} \in \mathbb{T}^{(d)}$ and $i \in[d]$, we let $\mathbf{t}^{(i)}=\left\{u \in \mathbf{t}: e_{\mathbf{t}}(u)=i\right\}$ be the set of vertices on $\mathbf{t}$ bearing the type $i$, and $\mathbf{f}^{(i)}$ the corresponding notation for the forest $\mathbf{f} \in \mathbb{F}^{(d)}$.

1.2. Multitype offspring distributions. We set $\mathbb{Z}_{+}=\{0,1,2, \ldots\}$ and $d \in \mathbb{N}$. A $d$-type offspring distribution $\boldsymbol{\mu}=\left(\mu^{(1)}, \ldots, \mu^{(d)}\right)$ is a family of distributions on the space $\mathbb{Z}_{+}^{d}$ of integer-valued non-negative sequences of length $d$. It will be useful to 
introduce the Laplace transforms $\varphi=\left(\varphi^{(1)}, \ldots, \varphi^{(d)}\right)$ of $\boldsymbol{\mu}$ by

$$
\varphi^{(i)}(\mathbf{s})=\sum_{\mathbf{z} \in \mathbb{Z}_{+}^{d}} \mu^{(i)}(\{\mathbf{z}\}) \exp (-\langle\mathbf{z}, \mathbf{s}\rangle), \quad \text { for } i \in[d],
$$

where $\mathbf{s}=\left(s_{1}, \ldots, s_{d}\right) \in \mathbb{R}_{+}^{d}$ and $\langle x, y\rangle$ is the usual scalar product of two vectors $x, y \in \mathbb{R}^{d}$. We let $\mathbf{0}$ be the vector of $\mathbb{R}_{+}^{d}$ with all components equal to 0 . Then, for $i, j \in[d]$, we define the quantity

$$
m_{i j}=-\frac{\partial \varphi^{(i)}}{\partial s_{j}}(\mathbf{0})=\sum_{\mathbf{z} \in \mathbb{Z}_{+}^{d}} z_{j} \mu^{(i)}(\{\mathbf{z}\})
$$

that corresponds to the mean number of children of type $j$, given by an individual of type $i$. We let $\mathbf{M}:=\left(m_{i j}\right)_{i, j \in[d]}$ be the mean matrix of $\boldsymbol{\mu}$, and $\mathbf{m}_{i}=$ $\left(m_{i 1}, \ldots, m_{i d}\right) \in \mathbb{R}_{+}^{d}$ be the mean vector of the measure $\mu^{(i)}$.

We say that a measure $\boldsymbol{\mu}$ on $\mathbb{Z}_{+}^{d}$ is non-degenerate, if there exists at least one $i \in[d]$ so that

$$
\mu^{(i)}\left(\left\{\mathbf{z} \in \mathbb{Z}_{+}^{d}: \sum_{j=1}^{d} z_{j} \neq 1\right\}\right)>0 .
$$

The offspring distribution that we consider in this work is assumed to be nondegenerate in order to avoid cases which will lead to infinite linear trees.

Definition 1.1. The mean matrix (or the offspring distribution $\boldsymbol{\mu}$ ) is called irreducible, if for every $i, j \in[d]$, there is some $n \in \mathbb{N}$ so that $m_{i j}^{(n)}>0$, where $m_{i j}^{(n)}$ is the $i j$-entry of the matrix $\mathbf{M}^{n}$.

Recall also that if $\mathbf{M}$ is irreducible, then according to the Perron-Frobenius theorem, $\mathbf{M}$ admits a unique eigenvalue $\rho$ which is simple, positive and with maximal modulus. Furthermore, the corresponding right and left eigenvectors can be chosen positive and we call them $\mathbf{a}=\left(a_{1}, \ldots, a_{d}\right)$ and $\mathbf{b}=\left(b_{1}, \ldots, b_{d}\right)$ respectively, and normalize them such that $\langle\mathbf{a}, 1\rangle=\langle\mathbf{a}, \mathbf{b}\rangle=1$; see Chapter $\mathrm{V}$ of Athreya and Ney (2004). We then say that $\boldsymbol{\mu}$ is sub-critical if $\rho<1$, critical $\rho=1$ and supercritical if $\rho>1$.

Main assumptions. Throughout this work, we consider an offspring distribution $\boldsymbol{\mu}=\left(\mu^{(1)}, \ldots, \mu^{(d)}\right)$ on $\mathbb{Z}_{+}^{d}$ satisfying the following conditions:

$\left(\mathbf{H}_{1}\right) \boldsymbol{\mu}$ is irreducible, non-degenerate and critical.

$\left(\mathbf{H}_{2} . \mathbf{1}\right)$ Let $\Delta$ be a nonempty subset of $[d]$. For every $i \in \Delta$, there exists $\alpha_{i} \in(1,2]$ such that the Laplace transform of $\mu^{(i)}$ satisfies

$$
\psi^{(i)}(\mathbf{s}):=-\log \varphi^{(i)}(\mathbf{s})=\left\langle\mathbf{m}_{i}, \mathbf{s}\right\rangle+|\mathbf{s}|^{\alpha_{i}} \Theta^{(i)}(\mathbf{s} /|\mathbf{s}|)+o\left(|\mathbf{s}|^{\alpha_{i}}\right), \quad \text { as }|\mathbf{s}| \downarrow 0,
$$

for $\mathbf{s} \in \mathbb{R}_{+}^{d}$ and where

$$
\Theta^{(i)}(\mathbf{s})=\int_{\mathbf{S}^{d}}|\langle\mathbf{s}, \mathbf{y}\rangle|^{\alpha_{i}} \lambda_{i}(\mathrm{~d} \mathbf{y}),
$$

with $\lambda_{i}$ a finite Borel non-zero measure on $\mathbf{S}^{d}=\left\{\mathbf{y} \in \mathbb{R}^{d}:|\mathbf{y}|=1\right\}$ such that for $\alpha_{i} \in(1,2), \lambda_{i}$ has support in $\left\{\mathbf{y} \in \mathbb{R}_{+}^{d}:|\mathbf{y}|=1\right\}$. We write $|\cdot|$ for the Euclidean norm. 
$\left(\mathbf{H}_{2} . \mathbf{2}\right)$ For $i \in[d] \backslash \Delta$, the Laplace transform of $\mu^{(i)}$ satisfies

$$
\psi^{(i)}(\mathbf{s}):=-\log \varphi^{(i)}(\mathbf{s})=\left\langle\mathbf{m}_{i}, \mathbf{s}\right\rangle+o\left(|\mathbf{s}|^{\alpha_{i}}\right), \quad \text { as }|\mathbf{s}| \downarrow 0 .
$$

where $\alpha_{i}=\min _{j \in \Delta} \alpha_{j}$ (which it does not depend on $i$ ).

Let us comment on these assumptions:

1. We notice that criticality, hypothesis $\left(\mathbf{H}_{1}\right)$, implies finiteness of all coefficients of the mean matrix $\mathbf{M}$.

2. For $i \in[d]$, we say that $\mu^{(i)}$ has finite variance when

$$
\frac{\partial^{2} \varphi^{(i)}}{\partial s_{j} \partial s_{k}}(\mathbf{0})<\infty, \quad \text { for } j, k \in[d] .
$$

We then write $\mathbf{Q}^{(i)}$ for its covariance matrix. In particular, when $\mu^{(i)}$ satisfies the condition $\left(\mathbf{H}_{2} . \mathbf{1}\right)$ with $\alpha_{i}=2$, one can easily verify that it possess finite variance and that it does not have variance when $\alpha_{i} \in(1,2)$. This shows that our assumptions on the offspring distribution are less restrictive than the ones made in Miermont (2008), where the author assumes finitess of the covariance matrices.

3. In the case when $\mu^{(i)}$ has finite variance, one can consider a measure $\lambda_{i}$ on $\mathbf{S}^{d}$ such that

$$
\Theta^{(i)}(\mathbf{s})=\left\langle\mathbf{s}, \mathbf{Q}^{(i)} \mathbf{s}\right\rangle, \quad \mathbf{s} \in \mathbb{R}_{+}^{d} ;
$$

see for example Section 2.4 of Samorodnitsky and Taqqu (1994).

4. Let $\boldsymbol{\xi}_{1}, \boldsymbol{\xi}_{2}, \ldots$ be a sequence of i.i.d. random variables on $\mathbb{Z}_{+}^{d}$ with common distribution $\mu^{(i)}$ satisfying $\left(\mathbf{H}_{2} \cdot \mathbf{1}\right)$. We observe that

$$
-\log \mathbb{E}\left[\exp \left(-\left\langle\frac{1}{n^{1 / \alpha_{i}}} \sum_{k=1}^{n}\left(\boldsymbol{\xi}_{k}-\mathbf{m}_{i}\right), \mathbf{s}\right\rangle\right)\right] \underset{n \rightarrow \infty}{\rightarrow}|\mathbf{s}|^{\alpha_{i}} \Theta^{(i)}(\mathbf{s} /|\mathbf{s}|),
$$

for $\mathbf{s} \in \mathbb{R}_{+}^{d}$. Then, we conclude that

$$
\frac{1}{n^{1 / \alpha_{i}}} \sum_{k=1}^{n}\left(\boldsymbol{\xi}_{k}-\mathbf{m}_{i}\right) \underset{n \rightarrow \infty}{\stackrel{d}{\longrightarrow}} \mathbf{Y}_{\alpha_{i}}
$$

where the convergence is in distribution and $\mathbf{Y}_{\alpha_{i}}$ is a $\alpha_{i}$-stable random vector in $\mathbb{R}_{+}^{d}$ whose Laplace exponent satisfies

$$
\psi_{\mathbf{Y}_{\alpha_{i}}}(\mathbf{s})=|\mathbf{s}|^{\alpha_{i}} \Theta^{(i)}(\mathbf{s} /|\mathbf{s}|), \quad \mathbf{s} \in \mathbb{R}_{+}^{d} .
$$

Satos book Sato (2013) and Samorodnitsky and Taqqu (1994) are good references for background on multivariate stable distributions. On the other hand, we notice from (1.1) that the equation (1.2) is equivalent to the hypothesis $\left(\mathbf{H}_{2} . \mathbf{1}\right)$.

5. We point out that in the monotype case, that is $d=1$, the condition $\left(\mathbf{H}_{2} . \mathbf{1}\right)$ may be thought as a particular case of the assumption made in Duquesne (2003) and Kortchemski (2013), which deal with arbitrary critical offspring distributions in the domain of attraction of an one-dimensional stable law, in order to get the convergence of the rescaled monotype GW tree to the continuum stable tree. 
6. For $i \in[d] \backslash \Delta$, let $\mu^{(i)}$ be a measure that satisfies the hypothesis $\left(\mathbf{H}_{2} . \mathbf{2}\right)$. We can rewrite the expression of its Laplace exponent in the following way

$$
\psi^{(i)}(\mathbf{s}):=-\log \varphi^{(i)}(\mathbf{s})=\left\langle\mathbf{m}_{i}, \mathbf{s}\right\rangle+|\mathbf{s}|^{\alpha_{i}} \Theta^{(i)}(\mathbf{s} /|\mathbf{s}|)+o\left(|\mathbf{s}|^{\alpha_{i}}\right),
$$

as $|\mathbf{s}| \downarrow 0$ and where

$$
\Theta^{(i)}(\mathbf{s})=\int_{\mathbf{S}^{d}}|\langle\mathbf{s}, \mathbf{y}\rangle|^{\alpha_{i}} \lambda_{i}(\mathrm{~d} \mathbf{y}),
$$

with $\lambda_{i} \equiv 0$. Recall that $\alpha_{i}=\min _{j \in \Delta} \alpha_{j}$ for $i \in[d] \backslash \Delta$. This will be useful for the rest of the work.

Finally, let $\underline{\alpha}=\min _{i \in[d]} \alpha_{i}$ and $\bar{\lambda}=\sum_{i \in[d]} \mathbb{1}_{\left\{\underline{\alpha}=\alpha_{i}\right\}} a_{i} \lambda_{i}$. We define

$$
\bar{c}=(\langle\mathbf{a}, \boldsymbol{\Theta}(\mathbf{b})\rangle)^{1 / \underline{\alpha}}=\left(\int_{\mathbf{S}^{d}}|\langle\mathbf{b}, \mathbf{y}\rangle|^{\underline{\alpha}} \bar{\lambda}(\mathrm{d} \mathbf{y})\right)^{1 / \underline{\alpha}},
$$

where $\boldsymbol{\Theta}(\mathbf{s})=\left(\Theta^{(1)}(\mathbf{s}) \mathbb{1}_{\left\{\underline{\alpha}=\alpha_{1}\right\}}, \ldots, \Theta^{(d)}(\mathbf{s}) \mathbb{1}_{\left\{\underline{\alpha}=\alpha_{d}\right\}}\right) \in \mathbb{R}_{+}^{d}$, for $\mathbf{s} \in \mathbb{R}_{+}^{d}$. We notice that $\bar{c} \not \equiv 0$ due to $\left(\mathbf{H}_{2} \cdot \mathbf{1}\right)$. This constant will play a role similar to the constant defined in equation (2) of Miermont (2008), i.e., it corresponds to the total variance of the offspring distribution $\boldsymbol{\mu}$, when the covariance matrices are finite.

1.3. Multitype Galton-Watson trees and forests. Let $\boldsymbol{\mu}$ be a $d$-type offspring distribution. We define the law $\mathbf{P}_{\boldsymbol{\mu}}^{(i)}$ (or simply $\mathbf{P}^{(i)}$ ) of a $d$-type GW tree (or multitype GW tree) rooted at a vertex of type $i \in[d]$ and with offspring distribution $\boldsymbol{\mu}$ by

$$
\mathbf{P}^{(i)}(T=\mathbf{t})=\prod_{u \in \mathbf{t}} \frac{c_{\mathbf{t}}^{(1)}(u) ! \ldots c_{\mathbf{t}}^{(d)}(u) !}{c_{\mathbf{t}}(u) !} \mu^{\left(e_{\mathbf{t}}(u)\right)}\left(\left\{c_{\mathbf{t}}^{(d)}(u), \ldots, c_{\mathbf{t}}^{(d)}(u)\right\}\right),
$$

where $T: \mathbb{T}^{(d)} \rightarrow \mathbb{T}^{(d)}$ is the identity map (see e.g., Abraham and Delmas, 2015, or Miermont, 2008 for a formal construction of a probability measure on $\left.\mathbb{T}^{(d)}\right)$. In particular, under the criticality assumption, $\left(\mathbf{H}_{1}\right)$, the multitype $\mathrm{GW}$ trees with offspring distribution $\boldsymbol{\mu}$ are almost surely finite. Similarly, for $\mathbf{x}=\left(x_{1}, \ldots, x_{r}\right)$ a finite sequence with terms in $[d]$, we define $\mathbf{P}_{\boldsymbol{\mu}}^{\mathbf{x}}$ (or simply $\mathbf{P}^{\mathbf{x}}$ ) the law of multitype $\mathrm{GW}$ forest with roots of type $\mathbf{x}$ and with offspring distribution $\boldsymbol{\mu}$ as the image measure of $\bigotimes_{j=1}^{r} \mathbf{P}^{\left(x_{j}\right)}$ by the map

$$
\left(\mathbf{t}_{(1)}, \ldots, \mathbf{t}_{(r)}\right) \longmapsto \cup_{k=1}^{r} k \mathbf{t}_{(k)},
$$

i.e., it is the law that makes the identity map $F: \mathbb{F}^{(d)} \rightarrow \mathbb{F}^{(d)}$ the random forest whose trees components $F_{1}, \ldots, F_{r}$ are independent with respective laws $\mathbf{P}^{\left(x_{1}\right)}, \ldots, \mathbf{P}^{\left(x_{d}\right)}$. A similar definition holds for an infinite sequence $\mathbf{x} \in[d]^{\mathbb{N}}$.

We then say that a $\mathbb{F}^{(d)}$-value random variable $F$ is a multitype $\mathrm{GW}$ forest with offspring distribution $\boldsymbol{\mu}$ and roots of type $\mathbf{x}$ when it has law $\mathbf{P}^{\mathbf{x}}$. Similarly, a $\mathbb{T}^{(d)}$-value random variable $T$ with law $\mathbf{P}^{(i)}$ is a multitype GW tree with offspring distribution $\boldsymbol{\mu}$ and root of type $i \in[d]$.

1.4. Main results. In this section, we state our main results on the asymptotic behavior of $d$-type GW trees with offspring distribution satisfying our main assumptions. In this direction, we first recall the definition of the discrete height process associated with a forest $\mathbf{f} \in \mathbb{F}$.

Let us denote by $\# \mathbf{t}$ the total progeny (or the total number of vertices) of $\mathbf{t}$. Similarly, \#f represents the total progeny of the forest $\mathbf{f}$. Let $\varnothing=u_{\mathbf{t}}(0) \prec u_{\mathbf{t}}(1) \prec$ 
$\ldots \prec u_{\mathbf{t}}(\# \mathbf{t}-1)$ be the list of vertices of $\mathbf{t}$ in depth-first order. The height process $H^{\mathbf{t}}=\left(H_{n}^{\mathbf{t}}, n \geq 0\right)$ is defined by $H_{n}^{\mathbf{t}}=\left|u_{\mathbf{t}}(n)\right|$, with the convention that $H_{n}^{\mathbf{t}}=0$ for $n \geq \# \mathbf{t}$. For the forest $\mathbf{f}$, we let $1=u_{\mathbf{f}}(0) \prec u_{\mathbf{f}}(1) \prec \cdots \prec u_{\mathbf{f}}(\# \mathbf{f}-1)$ be the depthfirst ordered list of its vertices, and write $H^{\mathbf{f}}=\left(H_{n}^{\mathbf{f}}, n \geq 0\right)$ by $H_{n}^{\mathbf{f}}=\left|u_{\mathbf{f}}(n)\right|-1$, for $0 \leq n<\# \mathbf{f}$. Detailed description and properties of this object can be found for example in Duquesne (2003).

Recall that $\underline{\alpha}=\min _{i \in[d]} \alpha_{i}$ with $\alpha_{1}, \ldots, \alpha_{d}$ as our main assumptions. Let $Y^{(\underline{\alpha})}=$ $\left(Y_{s}, s \geq 0\right)$ be a strictly stable spectrally positive Lévy process with index $\underline{\alpha} \in(1,2]$ with Laplace exponent

$$
\mathbb{E}\left[\exp \left(-\lambda Y_{s}\right)\right]=\exp \left(-s \lambda^{\underline{\alpha}}\right)
$$

for $\lambda \in \mathbb{R}_{+}$.

We can now state our main result.

Theorem 1.2. Let $F$ be a d-type $G W$ forest distributed according to $\mathbf{P}^{\mathbf{x}}$, for some arbitrary $\mathbf{x} \in[d]^{\mathbb{N}}$. Then, under $\mathbf{P}^{\mathbf{x}}$, the following convergence in distribution holds for the Skorohod topology on the space $\mathbb{D}\left(\mathbb{R}_{+}, \mathbb{R}\right)$ of right-continuous functions with left limits:

$$
\left(\frac{1}{n^{1-1 / \underline{\alpha}}} H_{\lfloor n s\rfloor}^{F}, s \geq 0\right) \underset{n \rightarrow \infty}{\stackrel{d}{\longrightarrow}}\left(\frac{1}{\bar{c}} H_{s}, s \geq 0\right),
$$

where $H$ stands for the continuous-time height process associated with the strictly stable spectrally positive Lévy process $Y^{(\underline{\alpha})}$.

In particular, we notice that this result implies the convergence in law of the $d$-type GW forest properly rescaled towards the stable forest of index $\underline{\alpha}$ for the Gromov-Hausdorff topology; see for example Lemma 2.4 of Le Gall (2005). On the other hand, when $\underline{\alpha}=2$, it is well-known that $\left(H_{s}, s \geq 0\right)$ is proportional to the reflected Brownian motion. The notion of height process for spectrally positive Lévy process has been studied in great detail in Duquesne and Le Gall (2002).

Next, for $n \geq 0$, we let $\Upsilon_{n}^{\mathbf{f}}$ be the first letter of $u_{\mathbf{f}}(n)$, with the convention that for $n \geq \# \mathbf{f}$, it equals the number of components of $\mathbf{f}$. In words, $\Upsilon_{n}^{\mathbf{f}}$ is the index of the tree component to which $u_{\mathbf{f}}(n)$ belongs.

Theorem 1.3. For $i \in[d]$, let $F$ be a d-type $G W$ forest distributed according to $\mathbf{P}^{\mathbf{i}}$, where $\mathbf{i}=(i, i, \ldots)$. Then, under $\mathbf{P}^{\mathbf{i}}$, we have the following convergence in distribution in $\mathbb{D}\left(\mathbb{R}_{+}, \mathbb{R}\right)$ :

$$
\left(\frac{1}{n^{1-1 / \underline{\alpha}}} \Upsilon_{\lfloor n s\rfloor}^{F}, s \geq 0\right) \underset{n \rightarrow \infty}{\stackrel{d}{\longrightarrow}}\left(-\frac{\bar{c}}{b_{i}} I_{s}, s \geq 0\right),
$$

where $I_{s}$ is the infimum at time sof the strictly stable spectrally positive Lévy process $Y^{(\underline{\alpha})}$.

Let us explain our approach while we describe the organization for the rest of the paper. We begin by exposing in Section 2.1 the key ingredient, that is, a remarkable decomposition of $d$-type forests into monotype forests. The plan then is to compare the corresponding height processes of the multitype GW forest and the monotype GW forest, and show that they are close for the Skorohod topology. In this direction, we will need to control the shape of large $d$-type GW forests. First, we establish in Section 2.2 sub-exponential tail bounds for the height and the number of tree components of $d$-type GW forests that may be of independent interest. Secondly, we estimate in Section 2.3 the asymptotic distribution of the 
different vertices types. To be a little more precise, Proposition 2.7 provides a convergence of types theorem for multitype GW trees, which extends Theorem 1 (iii) in Miermont (2008), for the infinite variance case. Roughly speaking, it shows that all types are homogeneously distributed in the limiting tree. We conclude with the proofs of Theorem 1.2 and 1.3 in Section 3 by pulling back the known results of Duquesne and Le Gall (2002) on the convergence of the rescaled height process of monotype GW forests to the multitype GW forest. Finally, in Section 4, we present two applications. The first one is an immediate consequence of Theorem 1.2 and 1.3 which provides information about the maximal height of a vertex in a multitype GW tree. Our second application involves a particular multitype GW tree, known as alternating two-type GW tree which appears frequently in the study of random planar maps. We establish a conditioned version of Theorem 1.2 for this special tree.

The global structure of the proofs is close to that Miermont (2008). Although we will try to make this work as self-contained as possible, we will often refer the reader to this paper when the proofs are readily adaptable, and will rather focus on the new technical ingredients. One difficulty arises from the fact that we are assuming weaker assumptions on the offspring distribution than in Miermont (2008), we do not assume a finitess of the covariances matrices of the offspring distributions and this forces us to improve some of Miermont's estimates.

\section{Preliminary results}

Through this section unless we specify otherwise, we let $F$ be $d$-type GW forest with law $\mathbf{P}^{\mathbf{x}}$ where $\mathbf{x} \in[d]^{\mathbb{N}}$ and such that its offspring distribution $\boldsymbol{\mu}=$ $\left(\mu^{(1)}, \ldots, \mu^{(d)}\right)$ satisfies the main assumptions. More precisely, it is important to keep in mind that there is a nonempty subset $\Delta$ of $[d]$ such that the family of distributions $\left(\mu^{(i)}\right)_{i \in \Delta}$ satisfy $\left(\mathbf{H}_{2} . \mathbf{1}\right)$ while the remainder $\left(\mu^{(i)}\right)_{i \in[d] \backslash \Delta}$ fulfills $\left(\mathbf{H}_{2} . \mathbf{2}\right)$.

2.1. Decomposition of multitype $G W$ forests. In this section, we introduce the projection function $\Pi^{(i)}$ defined by Miermont (2008) that goes from the set of $d$-types planar forests to the set of monotype planar forests. Roughly speaking, the function $\Pi^{(i)}$ removes all the vertices of type different from $i$ and then it connects the remaining vertices with their most recent common ancestor, preserving the lexicographical order. More precisely, set a $d$-type forest $\mathbf{f} \in \mathbb{F}^{d}$ and let $v_{1} \prec v_{2} \prec \cdots$ be the vertices of $\mathbf{f}^{(i)}$ listed in depth-first order such that all ancestors of $v_{k}$ have types different from $i$. They will be the roots of the new forest. We then build a forest $\Pi^{(i)}(\mathbf{f})=\mathbf{f}^{\prime}$ with as many tree components as there are elements in $\left\{v_{1}, v_{2}, \ldots\right\}$. Recursively, starting from the set of roots $1,2, \ldots$ of $\mathbf{f}^{\prime}$, for each $u \in \mathbf{f}^{\prime}$, we let $v_{u 1}, v_{u 2}, \ldots, v_{u k}$ be vertices of $\left(v_{u} \mathbf{f}_{v_{u}}\right) \backslash\left\{v_{u}\right\}$ arranged in lexicographical order and such that:

I. They have type $i$, i.e. $e_{\mathbf{f}}\left(v_{u j}\right)=i$ for $1 \leq j \leq k$,

II. All their ancestors on $\left(v_{u} \mathbf{f}_{v_{u}}\right) \backslash\left\{v_{u}\right\}$ have types different from $i$ (if any).

Then, we add the vertices $u 1, \ldots, u k$ to $\mathbf{f}^{\prime}$ as children of $u$, and continue iteratively. See Figure 2.1 for an example when $d=3$. 


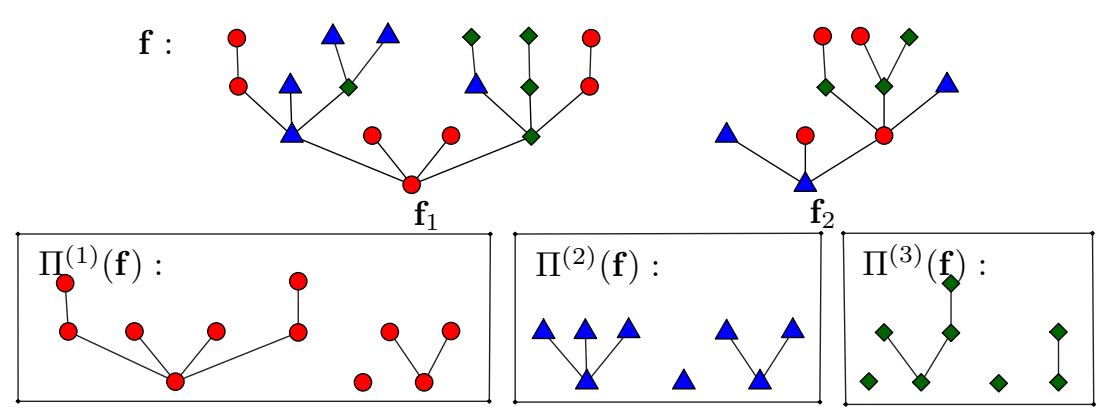

Figure 2.1. A realization of the projection $\Pi^{(i)}$ for a three-type planar forest with two tree components, type 1 vertices represented with circles, type 2 vertices with triangles and type 3 vertices with diamonds.

We have the following key result:

Proposition 2.1. Let $\mathbf{x} \in[d]^{\mathbb{N}}$ and $i \in[d]$. Then, under the law $\mathbf{P}^{\mathbf{x}}$, the forest $\Pi^{(i)}(F)$ is a monotype $G W$ forest with critical non-degenerate offspring distribution $\bar{\mu}^{(i)}$ that is in the domain of attraction of a stable law of index $\underline{\alpha}=\min _{j \in[d]} \alpha_{j}$. More precisely, the Laplace exponent of $\bar{\mu}^{(i)}$ satisfies

$$
\bar{\psi}^{(i)}(s)=s+\frac{1}{a_{i}}\left(\frac{\bar{c}}{b_{i}} s\right)^{\underline{\alpha}}+o\left(s^{\underline{\alpha}}\right), \quad s \downarrow 0,
$$

where $s \in \mathbb{R}_{+}$.

The proof of this proposition is based in an inductive argument that consists in removing types one by one until we are left with a monotype GW forests. More precisely, we suppose that the vertices with type $d$ are removed from the forest f $\in \mathbb{F}^{(d)}$. We point out that one can delete any other type similarly. We let $v_{1} \prec v_{2} \prec \ldots$ be the vertices of $\mathbf{f}$ listed in depth-first order such that $e_{\mathbf{f}}\left(v_{i}\right) \neq d$ and $e_{\mathbf{f}}(v)=d$ for every $v \vdash v_{i}$. These are the vertices of $\mathbf{f}$ with type different from $d$ which do not have ancestors of type $d$. We build a forest $\tilde{\Pi}(\mathbf{f})=\tilde{\mathbf{f}}$ recursively. We start from the set $\left\{v_{1}, v_{2}, \ldots\right\}$ and for each $v_{u} \in \tilde{\mathbf{f}}$, we let $v_{u 1} \prec \cdots \prec v_{u k}$ be the descendants of $v_{u}$ in $\mathbf{f}$ such that:

I. They have type different from $d$.

II. For $1 \leq j \leq k$, all the vertices between $v_{u}$ and $v_{u j}$ have type $d$ (if any).

Then, we add these vertices to $\tilde{\mathbf{f}}$, and continue in an obvious way. We naturally associate the type $e_{\mathbf{f}}$ to the vertices of $\tilde{\Pi}(\mathbf{f})$. In the sequel, we refer to this procedure as the $\mathbf{d}$ - to $(\mathbf{d}-\mathbf{1})$-type operation.

The following lemma shows that after performing the $d$-to $(d-1)$-type operation in the multitype $\mathrm{GW}$ forest $F$, we obtain a $(d-1)$-type $\mathrm{GW}$ forest which offspring distribution still satisfying our main assumptions. First, we fix some notation. We denote by $\tilde{\mathbf{m}}_{d}$ the vector in $\mathbb{R}_{+}^{d-1}$ with entries

$$
\tilde{m}_{d k}=\frac{m_{d k}}{1-m_{d d}}, \quad \text { for } k \in[d-1],
$$


and for $j \in[d-1]$, we write $\tilde{\mathbf{m}}_{j}$ for the vector in $\mathbb{R}_{+}^{d-1}$ with entries

$$
\tilde{m}_{j k}=m_{j k}+\frac{m_{j d} m_{d k}}{1-m_{d d}}, \quad \text { for } k \in[d-1] .
$$

We stress that due to the irreducibility assumption on the mean matrix $\mathbf{M}$ of the measure $\boldsymbol{\mu}$, we have that $1-m_{j j}>0$ for all $j \in[d]$. Thus, all the previous quantities are finite.

Lemma 2.2. Let $\mathbf{x} \in[d]^{\mathbb{N}}$. Then, under the law $\mathbf{P}^{\mathbf{x}}$, the forest $\tilde{\Pi}(F)$ is a nondegenerate, irreducible, critical $(d-1)$-type $G W$ forest. Moreover, its offspring distribution $\tilde{\boldsymbol{\mu}}=\left(\tilde{\mu}^{(1)}, \ldots, \tilde{\mu}^{(d-1)}\right)$ has Laplace exponents

$$
\tilde{\psi}^{(j)}(\mathbf{s})=\left\langle\tilde{\mathbf{m}}_{j}, \mathbf{s}\right\rangle+|\mathbf{s}|^{\tilde{\alpha}_{j}} \tilde{\Theta}^{(j)}(\mathbf{s} /|\mathbf{s}|)+o\left(|\mathbf{s}|^{\tilde{\alpha}_{j}}\right), \quad|\mathbf{s}| \downarrow 0,
$$

for $j \in[d-1], \mathbf{s} \in \mathbb{R}_{+}^{d-1}, \tilde{\alpha}_{j}=\min \left(\alpha_{j}, \alpha_{d}\right)$ and

$$
\tilde{\Theta}^{(j)}(\mathbf{s})=\int_{\mathbf{S}^{d}}\left|\left\langle\mathbf{s}, \tilde{\mathbf{y}}+y_{d} \tilde{\mathbf{m}}_{d}\right\rangle\right|^{\tilde{\alpha}_{j}} \tilde{\lambda}_{j}(\mathrm{~d} \mathbf{y}),
$$

where $\tilde{\lambda}_{j}=\mathbb{1}_{\left\{\tilde{\alpha}_{j}=\alpha_{j}\right\}} \lambda_{j}+\mathbb{1}_{\left\{\tilde{\alpha}_{j}=\alpha_{d}\right\}} \frac{m_{j d}}{1-m_{d d}} \lambda_{d}, \mathbf{y}=\left(y_{1}, \ldots, y_{d}\right) \in \mathbb{R}^{d}$ and $\tilde{\mathbf{y}}=$ $\left(y_{1}, \ldots, y_{d-1}\right) \in \mathbb{R}^{d-1}$.

It is important to stress that $\tilde{\lambda}_{j} \equiv 0$ when $j, d \in[d] \backslash \Delta$, and otherwise it is non-zero (recall the last comment after the introduction of the main assumptions in Section 1.2).

Proof: The fact that $\tilde{\Pi}(F)$ is a non-degenerate, irreducible, critical $(d-1)$-type GW forest follows from Lemma 3 (i) in Miermont (2008). Moreover, we deduce from this same lemma (see specifically equations (8) and (9) in Miermont, 2008) that the offspring distribution $\tilde{\boldsymbol{\mu}}=\left(\tilde{\mu}^{(1)}, \ldots, \tilde{\mu}^{(d-1)}\right)$ has Laplace exponents

$$
\tilde{\psi}^{(j)}(\mathbf{s})=\psi^{(j)}\left(\mathbf{s}, \tilde{\psi}^{(d)}(\mathbf{s})\right),
$$

for $j \in[d-1]$ and $\mathbf{s} \in \mathbb{R}_{+}^{d-1}$, where $\tilde{\psi}^{(d)}$ is implicitly defined by

$$
\tilde{\psi}^{(d)}(\mathbf{s})=\psi^{(d)}\left(\mathbf{s}, \tilde{\psi}^{(d)}(\mathbf{s})\right) \text {. }
$$

This is obtained by separating the offspring of each individual with types equal and different from $d$.

In order to understand the behavior of $\tilde{\psi}^{(j)}$ close to zero, we start by analyzing the one of $\tilde{\psi}^{(d)}$. In this direction, we observe from (2.2) and our main assumptions on the offspring distribution $\boldsymbol{\mu}$ that

$$
\begin{aligned}
\tilde{\psi}^{(d)}(\mathbf{s})=(1- & \left.m_{d d}\right)\left\langle\tilde{\mathbf{m}}_{d}, \mathbf{s}\right\rangle+m_{d d} \tilde{\psi}^{(d)}(\mathbf{s}) \\
& +\left|\left(\mathbf{s}, \tilde{\psi}^{(d)}(\mathbf{s})\right)\right|^{\alpha_{d}} \Theta^{(d)}\left(\frac{\left(\mathbf{s}, \tilde{\psi}^{(d)}(\mathbf{s})\right)}{\left|\left(\mathbf{s}, \tilde{\psi}^{(d)}(\mathbf{s})\right)\right|}\right)+o\left(\left|\left(\mathbf{s}, \tilde{\psi}^{(d)}(\mathbf{s})\right)\right|^{\alpha_{d}}\right) \\
= & \left\langle\tilde{\mathbf{m}}_{d}, \mathbf{s}\right\rangle+\frac{1}{1-m_{d d}}\left|\left(\mathbf{s}, \tilde{\psi}^{(d)}(\mathbf{s})\right)\right|^{\alpha_{d}} \Theta^{(d)}\left(\frac{\left(\mathbf{s}, \tilde{\psi}^{(d)}(\mathbf{s})\right)}{\left|\left(\mathbf{s}, \tilde{\psi}^{(d)}(\mathbf{s})\right)\right|}\right)+o\left(\left|\left(\mathbf{s}, \tilde{\psi}^{(d)}(\mathbf{s})\right)\right|^{\alpha_{d}}\right),
\end{aligned}
$$

as $|\mathbf{s}| \downarrow 0$. We also notice that

$$
\tilde{\psi}^{(d)}(\mathbf{s})=\left\langle\tilde{\mathbf{m}}_{d}, \mathbf{s}\right\rangle+o(|\mathbf{s}|), \quad \text { as }|\mathbf{s}| \downarrow 0 .
$$

On the one hand, from the above estimate, we know that

$$
\left\langle\left(\mathbf{s}, \tilde{\psi}^{(d)}(\mathbf{s})\right), \mathbf{y}\right\rangle=\left\langle\mathbf{s}, \tilde{\mathbf{y}}+y_{d} \tilde{\mathbf{m}}_{d}\right\rangle+y_{d} O(|\mathbf{s}|), \quad \text { as }|\mathbf{s}| \downarrow 0,
$$


Thus,

$$
\begin{aligned}
\left|\left(\mathbf{s}, \tilde{\psi}^{(d)}(\mathbf{s})\right)\right|^{\alpha_{d}} & \Theta^{(d)}\left(\frac{\left(\mathbf{s}, \tilde{\psi}^{(d)}(\mathbf{s})\right)}{\left|\left(\mathbf{s}, \tilde{\psi}^{(d)}(\mathbf{s})\right)\right|}\right) \\
& =\int_{\mathbf{S}^{d}}\left|\left\langle\left(\mathbf{s}, \tilde{\psi}^{(d)}(\mathbf{s})\right), \mathbf{y}\right\rangle\right|^{\alpha_{d}} \lambda_{d}(\mathrm{~d} \mathbf{y}) \\
& =\int_{\mathbf{S}^{d}}\left|\left\langle\mathbf{s}, \tilde{\mathbf{y}}+y_{d} \tilde{\mathbf{m}}_{d}\right\rangle\right|^{\alpha_{d}} \lambda_{d}(\mathrm{~d} \mathbf{y})+o\left(|\mathbf{s}|^{\alpha_{d}}\right)
\end{aligned}
$$

On the other hand, from (2.3), we have that

$$
\left\langle\left(\mathbf{s}, \tilde{\psi}^{(d)}(\mathbf{s})\right),\left(\mathbf{s}, \tilde{\psi}^{(d)}(\mathbf{s})\right)\right\rangle=\langle\mathbf{s}, \mathbf{s}\rangle+\left\langle\mathbf{s}, \tilde{\mathbf{m}}_{d}\right\rangle^{2}+o\left(|\mathbf{s}|^{2}\right), \quad \text { as }|\mathbf{s}| \downarrow 0 .
$$

Then, the previous estimates yield that

$$
\tilde{\psi}^{(d)}(\mathbf{s})=\left\langle\tilde{\mathbf{m}}_{d}, \mathbf{s}\right\rangle+\frac{1}{1-m_{d d}}|\mathbf{s}|^{\alpha_{d}} \tilde{\Theta}^{(d)}(\mathbf{s} /|\mathbf{s}|)+o\left(|\mathbf{s}|^{\alpha_{d}}\right), \quad|\mathbf{s}| \downarrow 0,
$$

where

$$
\tilde{\Theta}^{(d)}(\mathbf{s})=\int_{\mathbf{S}^{d}}\left|\left\langle\mathbf{s}, \tilde{\mathbf{y}}+y_{d} \tilde{\mathbf{m}}_{d}\right\rangle\right|^{\alpha_{d}} \lambda_{d}(\mathrm{~d} \mathbf{y}), \quad \text { for } \mathbf{s} \in \mathbb{R}_{+}^{d-1} \text {. }
$$

Next, we deduce the asymptotic behavior of $\tilde{\psi}^{(j)}$. It follows from (2.1) and our main assumption on the offspring distribution that

$$
\begin{aligned}
\tilde{\psi}^{(j)}(\mathbf{s})=\left\langle\tilde{\mathbf{m}}_{j}\right. & \left.-m_{j d} \tilde{\mathbf{m}}_{d}, \mathbf{s}\right\rangle+m_{j d} \tilde{\psi}^{(d)}(\mathbf{s}) \\
& +\left|\left(\mathbf{s}, \tilde{\psi}^{(d)}(\mathbf{s})\right)\right|^{\alpha_{j}} \Theta^{(j)}\left(\frac{\left(\mathbf{s}, \tilde{\psi}^{(d)}(\mathbf{s})\right)}{\left|\left(\mathbf{s}, \tilde{\psi}^{(d)}(\mathbf{s})\right)\right|}\right)+o\left(\left|\left(\mathbf{s}, \tilde{\psi}^{(d)}(\mathbf{s})\right)\right|^{\alpha_{j}}\right) \\
=\left\langle\tilde{\mathbf{m}}_{j}-m_{j d} \tilde{\mathbf{m}}_{d}, \mathbf{s}\right\rangle+m_{j d} \tilde{\psi}^{(d)}(\mathbf{s}) & \\
& +\left|\left(\mathbf{s}, \tilde{\psi}^{(d)}(\mathbf{s})\right)\right|^{\alpha_{j}} \Theta^{(j)}\left(\frac{\left(\mathbf{s}, \tilde{\psi}^{(d)}(\mathbf{s})\right)}{\left|\left(\mathbf{s}, \tilde{\psi}^{(d)}(\mathbf{s})\right)\right|}\right)+o\left(|\mathbf{s}|^{\alpha_{j}}\right)
\end{aligned}
$$

as $|\mathbf{s}| \downarrow 0$, where we have used (2.5) for the last equality. We observe that a similar computation as (2.4) shows that

$$
\left|\left(\mathbf{s}, \tilde{\psi}^{(d)}(\mathbf{s})\right)\right|^{\alpha_{j}} \Theta^{(j)}\left(\frac{\left(\mathbf{s}, \tilde{\psi}^{(d)}(\mathbf{s})\right)}{\left|\left(\mathbf{s}, \tilde{\psi}^{(d)}(\mathbf{s})\right)\right|}\right)=\int_{\mathbf{S}^{d}}\left|\left\langle\mathbf{s}, \tilde{\mathbf{y}}+y_{d} \tilde{\mathbf{m}}_{d}\right\rangle\right|^{\alpha_{j}} \lambda_{j}(\mathrm{~d} \mathbf{y})+o\left(|\mathbf{s}|^{\alpha_{j}}\right) .
$$

Consequently, combining the last display with (2.6), we deduce that equation (2.7) becomes for $|\mathbf{s}| \downarrow 0$,

$$
\begin{aligned}
\tilde{\psi}^{(j)}(\mathbf{s})=\left\langle\tilde{\mathbf{m}}_{j}, \mathbf{s}\right\rangle & +\frac{m_{j d}}{1-m_{d d}}|\mathbf{s}|^{\alpha_{d}} \tilde{\Theta}^{(d)}(\mathbf{s} /|\mathbf{s}|) \\
& +\int_{\mathbf{S}^{d}}\left|\left\langle\mathbf{s}, \tilde{\mathbf{y}}+y_{d} \tilde{\mathbf{m}}_{d}\right\rangle\right|^{\alpha_{j}} \lambda_{j}(\mathrm{~d} \mathbf{y})+o\left(|\mathbf{s}|^{\alpha_{d}}\right)+o\left(|\mathbf{s}|^{\alpha_{j}}\right)
\end{aligned}
$$

which clearly implies our claim.

We notice that after performing the $d$ - to $(d-1)$-type operation, we are left with a non-degenerate, irreducible, critical $(d-1)$-type GW forest whose offspring distribution $\tilde{\boldsymbol{\mu}}$ has mean matrix $\tilde{\mathbf{M}}=\left(\tilde{m}_{j k}\right)_{j, k \in[d-1]}$. Lemma 2.2 shows that this 
matrix has spectral radius 1 and moreover, it is not difficult to check that its left and right 1-eigenvectors $\tilde{\mathbf{a}}, \tilde{\mathbf{b}}$ satisfying $\langle\tilde{\mathbf{a}}, \mathbf{1}\rangle=\langle\tilde{\mathbf{a}}, \tilde{\mathbf{b}}\rangle=1$ are given by

$$
\tilde{\mathbf{a}}=\frac{1}{1-a_{d}}\left(a_{1}, \ldots, a_{d-1}\right) \quad \text { and } \quad \tilde{\mathbf{b}}=\frac{1-a_{d}}{1-a_{d} b_{d}}\left(b_{1}, \ldots, b_{d-1}\right) .
$$

We are now able to establish Proposition 2.1.

Proof of Proposition 2.1: The fact that $\Pi^{(i)}(F)$ is a monotype GW forest with critical non-degenerate offspring distribution is a consequence of Lemma 2.2 by following exactly the same argument as the proof of Proposition 4 (i) in Miermont (2008). Roughly speaking, the idea is to remove the types different from $i$ one by one through the $d$ - to $(d-1)$-type operation, and noticing that the hypotheses of the GW forest under consideration are conserved at every step until we are left with a critical non-degenerate monotype GW forest. Thus, it only remains to show that the offspring distribution of $\Pi^{(i)}(F)$ is in the domain of attraction of a stable law of index $\underline{\alpha}=\min _{j \in[d]} \alpha_{j}$. We prove this by induction on $d$, in the case $i=1$, without losing generality. The case $d=1$ is obvious. So suppose $d \geq 2$. We apply the $d$ to $(d-1)$-type operation through Lemma 2.2, and use the induction hipothesis to conclude that the offspring distribution $\bar{\mu}^{(1)}$ of $\Pi^{(1)}(F)$ satisfies

$$
\bar{\psi}^{(1)}(s)=s+\frac{1}{\tilde{a}_{1}}\left(\frac{\tilde{c}}{\tilde{b}_{1}}\right)^{\underline{\alpha}}+o\left(s^{\underline{\alpha}}\right), \quad s \downarrow 0,
$$

for $s \in \mathbb{R}_{+}$and where $\tilde{c}=(\langle\tilde{\mathbf{a}}, \tilde{\mathbf{\Theta}}(\tilde{\mathbf{b}})\rangle)^{1 / \underline{\alpha}}$, with

$$
\tilde{\mathbf{\Theta}}(\mathbf{s})=\left(\tilde{\Theta}^{(1)}(\mathbf{s}) \mathbb{1}_{\left\{\underline{\alpha}=\tilde{\alpha}_{1}\right\}}, \ldots, \tilde{\Theta}^{(d-1)}(\mathbf{s}) \mathbb{1}_{\left\{\underline{\alpha}=\tilde{\alpha}_{d-1}\right\}}\right),
$$

as in Lemma 2.2. On the other hand, we first observe that for $j \in[d-1]$, we have

$$
\begin{aligned}
\tilde{\Theta}^{(j)}(\tilde{\mathbf{b}}) & =\int_{\mathbf{S}^{d}}\left|\left\langle\tilde{\mathbf{b}}, \tilde{\mathbf{y}}+y_{d} \tilde{\mathbf{m}}_{d}\right\rangle\right|^{\tilde{\alpha}_{j}} \tilde{\lambda}_{j}(\mathrm{~d} \mathbf{y}) \\
& =\int_{\mathbf{S}^{d}}\left|\langle\tilde{\mathbf{b}}, \tilde{\mathbf{y}}\rangle+y_{d}\left(\tilde{\mathbf{b}}, \tilde{\mathbf{m}}_{d}\right\rangle\right|^{\tilde{\alpha}_{j}} \tilde{\lambda}_{j}(\mathrm{~d} \mathbf{y}) \\
& =\left(\frac{1-a_{d}}{1-a_{d} b_{d}}\right)^{\tilde{\alpha}_{j}} \int_{\mathbf{S}^{d}}\left|\sum_{k=1}^{d-1} b_{k} y_{k}+y_{d} \sum_{k=1}^{d-1} b_{k} \frac{m_{d k}}{1-m_{d d}}\right|^{\tilde{\alpha}_{j}} \tilde{\lambda}_{j}(\mathrm{~d} \mathbf{y}) \\
& =\left(\frac{1-a_{d}}{1-a_{d} b_{d}}\right)^{\tilde{\alpha}_{j}}\left(\Theta^{(j)}(\mathbf{b}) \mathbb{1}_{\left\{\tilde{\alpha}_{j}=\alpha_{j}\right\}}+\Theta^{(d)}(\mathbf{b}) \frac{m_{j d}}{1-m_{d d}} \mathbb{1}_{\left\{\tilde{\alpha}_{j}=\alpha_{d}\right\}}\right),
\end{aligned}
$$

where for the last equality, we use the fact the $\mathbf{b}$ is the right 1-eigenvector of the mean matrix $\mathbf{M}$, that is, $\sum_{k \in[d]} b_{k} m_{d k}=b_{d}$. Then, from the previous identity, we have that

$$
\begin{aligned}
& \langle\tilde{\mathbf{a}}, \tilde{\boldsymbol{\Theta}}(\tilde{\mathbf{b}})\rangle \\
& =\left(\frac{1-a_{d}}{1-a_{d} b_{d}}\right)^{\underline{\alpha}}\left(\sum_{k=1}^{d-1} \tilde{a}_{k} \Theta^{(k)}(\mathbf{b}) \mathbb{1}_{\left\{\underline{\alpha}=\alpha_{k}\right\}}+\Theta^{(d)}(\mathbf{b}) \mathbb{1}_{\left\{\underline{\alpha}=\alpha_{d}\right\}} \sum_{k=1}^{d-1} \tilde{a}_{k} \frac{m_{k d}}{1-m_{d d}}\right) \\
& =\frac{\left(1-a_{d}\right)^{\underline{\alpha}-1}}{\left(1-a_{d} b_{d}\right)^{\underline{\alpha}}}\langle\mathbf{a}, \boldsymbol{\Theta}(\mathbf{b})\rangle,
\end{aligned}
$$

where in the last equality, we now use that $\mathbf{a}$ is the left 1-eigenvector of the mean matrix M, i.e., $\sum_{k \in[d]} a_{k} m_{k d}=a_{d}$. Therefore, the fact that $\bar{\mu}^{(1)}$ belongs to the 
domain of attraction of a stable law of index $\underline{\alpha}$ follows by induction and the above equality.

Following Miermont (2008), we are interested in keeping the information of the number vertices that we delete during the projection $\Pi^{(i)}$. More precisely, for $\mathbf{f} \in \mathbb{F}^{(d)}$, recall that $\Pi^{(i)}(\mathbf{f})$ is the monotype forest obtained by removing all the vertices with type different from $i$. Then, for a vertex $u \in \Pi^{(i)}(\mathbf{f})$ with children $u 1, \ldots, u k$, we let $\mathbf{f}_{v_{u}}, \mathbf{f}_{v_{u 1}}, \ldots, \mathbf{f}_{v_{u k}}$ be the subtrees of the original forest $\mathbf{f}$ rooted at $u, u 1, \ldots, u k$, respectively. Then, we let

$$
N_{i j}(u)=\#\left\{w \in \mathbf{f}_{v_{u}} \backslash\left(\bigcup_{r=1}^{k} \mathbf{f}_{v_{u r}}\right): e_{\mathbf{f}}(w)=j\right\}, \quad \text { for } j \in[d] \backslash\{i\},
$$

be the number of type $j$ vertices that have been deleted between $u$ and its children. We also let

$$
\hat{N}_{i j}(n)=\#\left\{v \in \mathbf{f}_{n}: e_{\mathbf{f}}(v)=j \text { and } e_{\mathbf{f}}(w) \neq i \text { for all } w \vdash v\right\}, \quad \text { for } j \in[d] \backslash\{i\} \text {, }
$$

be the number of type $j$ vertices of the $n$-th tree component of $\mathbf{f}$ that lie below the first layer of type $i$ vertices, i.e. the number of type $j$ vertices of $\mathbf{f}_{n}$ that do not have ancestors of type $i$.
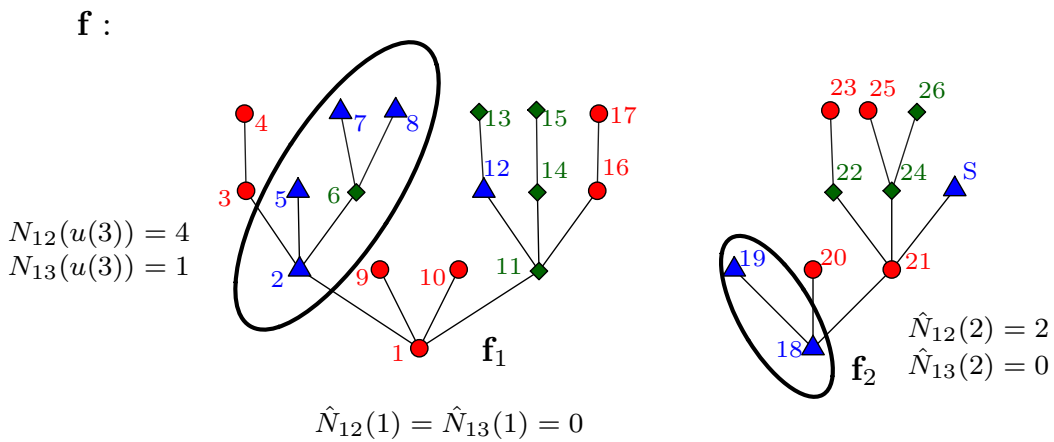

Figure 2.2. A representation of the quantities $N_{1 j}$ and $\hat{N}_{2 j}$, for a three-type planar forest with two tree components, type 1 vertices represented with circles, type 2 vertices with triangles and type 3 vertices with diamonds.

The following proposition provides information about the distribution of the previous quantities.

Proposition 2.3. Let $\varnothing=u(0) \prec u(1) \prec \cdots \prec u\left(\# \Pi^{(i)}(\mathbf{f})-1\right)$ be the list of vertices of $\Pi^{(i)}(\mathbf{f})$ in depth-first order and let $\mathbf{x} \in[d]^{\mathbb{N}}$. Then, under the law $\mathbf{P}^{\mathbf{x}}$ and for each $i \in[d]$ :

(i) For every $j \in[d] \backslash\{i\}$, the random variables $\left(N_{i j}(u(n)), n \geq 0\right)$ are i.i.d. Moreover, their Laplace exponents satisfy

$$
\phi_{i j}(s):=-\log \mathbf{E}^{\mathbf{x}}\left[\exp \left(-s N_{i j}(u(0))\right)\right]=\frac{a_{j}}{a_{i}} s+c_{i j} s^{\underline{\alpha}}+o\left(s^{\underline{\alpha}}\right),
$$


as $s \downarrow 0$, where $s \in \mathbb{R}_{+}, \underline{\alpha}=\min _{j \in[d]} \alpha_{j}$ and $c_{i j}>0$ a constant. In particular, $\mathbf{E}^{\mathbf{x}}\left[N_{i j}(u(0))\right]=a_{j} / a_{i}$.

(ii) For every $j \in[d] \backslash\{i\}$, the random variables $\left(\hat{N}_{i j}(n), n \geq 1\right)$ are independent, and their Laplace exponents satisfy

$$
\begin{aligned}
& \hat{\phi}_{i j}(s):=-\log \mathbf{E}^{\mathbf{x}}\left[\exp \left(-s \hat{N}_{i j}(n)\right)\right]=\left(\hat{c}_{i j} s+\hat{c}_{i j}^{\prime} s^{\hat{\alpha}_{i}}+o\left(s^{\hat{\alpha}_{i}}\right)\right) \mathbb{1}_{\left\{x_{n} \neq i\right\}}, \\
& \text { as } s \downarrow 0, \text { for } s \in \mathbb{R}_{+} \text {, some constants } \hat{c}_{i j}>0 \text { and } \hat{c}_{i j}^{\prime} \geq 0 \text { (that depends on } \\
& \left.x_{n}\right) \text { and where } \hat{\alpha}_{i}=\min _{j \in[d] \backslash\{i\}} \alpha_{j} .
\end{aligned}
$$

The idea of the proof is based in a similar induction argument as in the one of Proposition 2.1, by making use of the $d$ - to $(d-1)$-type operation $\tilde{\Pi}$. In this direction, we notice that the left and right 1-eigenvectors $\mathbf{a}, \mathbf{b}$ of $\mathbf{M}$ satisfy, for $1 \leq j \leq d$

$$
a_{j}=\sum_{i=1}^{d} a_{i} m_{i j} \quad \text { and } \quad b_{i}=\sum_{j=1}^{d} b_{j} m_{i j}
$$

for $1 \leq i \leq d$. In particular, when $d=2$, a simple computation shows that

$$
\frac{a_{2}}{a_{1}}=\frac{m_{12}}{1-m_{22}}=\frac{1-m_{11}}{m_{21}} \quad \text { and } \quad \frac{b_{2}}{b_{1}}=\frac{m_{21}}{1-m_{22}}=\frac{1-m_{11}}{m_{12}} .
$$

This will be useful in a moment.

Proof:

(i) The fact that for every $j \in[d] \backslash\{i\}$, the random variables $\left(N_{i j}(u(n)), n \geq 0\right)$ are i.i.d. has been proven in Proposition 4 (ii) of Miermont (2008). Basically, this follows from Jagers' theorem on stopping lines Jagers (1989). We then focus on the second part of the statement, and for simplicity, we prove this in the case $i=1$, without losing generality. The idea is based in a similar induction argument as in the proof of Proposition 2.1, by making use of the $d$ - to $(d-1)$-type operation $\tilde{\Pi}$. In this direction, for $\mathbf{f} \in \mathbb{F}^{(d)}$ and $u \in \tilde{\Pi}(\mathbf{f})$, we let $\tilde{N}(u)$ be the number of $d$-type vertices that have been deleted between $u$ and its children during this procedure. For $j \in[d-1]$, we let $u^{(j)}(0) \prec u^{(j)}(1) \prec \ldots$ be the type $j$ vertices of $F$ arranged in depth-first order. Then, Lemma 3 (ii) in Miermont (2008) ensures that under $\mathbf{P}^{\mathbf{x}}$, the $d-1$ sequences $\left(\tilde{N}\left(u^{(j)}(n)\right), n \geq 0\right)$ are independent and formed of i.i.d. elements. Further, their Laplace exponents $\tilde{\phi}^{(j)}$ respectively satisfy

$$
\tilde{\phi}^{(j)}(s)=\psi^{(j)}\left(\mathbf{0}, \tilde{\phi}^{(d)}(s)\right)
$$

for $s \in \mathbb{R}_{+}, \mathbf{0}$ the vector of $\mathbb{R}_{+}^{d-1}$ with all components equal to 0 , and where $\tilde{\phi}^{(d)}$ is implicitly given by

$$
\tilde{\phi}^{(d)}(s)=s+\psi^{(d)}\left(\mathbf{0}, \tilde{\phi}^{(d)}(s)\right)
$$

Thus, from our main assumptions on the offspring distribution, it is not difficult to check by following the same reasoning as the proof of Lemma 2.2 that

$$
\tilde{\phi}^{(j)}(s)=\frac{m_{j d}}{1-m_{d d}} s+\tilde{c}_{j d} s^{\tilde{\alpha}_{j}}+o\left(s^{\tilde{\alpha}_{j}}\right), \quad \text { as } s \downarrow 0,
$$

where $\tilde{\alpha}_{j}=\min \left(\alpha_{j}, \alpha_{d}\right)$ and the constant $\tilde{c}_{j d}=0$ if $j, d \in[d] \backslash \Delta$ and $\tilde{c}_{j d}>0$ otherwise (recall the main assumptions $\left(\mathbf{H}_{2} . \mathbf{1}\right)$ and $\left(\mathbf{H}_{2} . \mathbf{2}\right)$ ). 
Let now proceed to prove our statement. In the monotype case, $d=1$, there is nothing to show. For the case $d=2$, one checks from the previous discussion that the Laplace exponent of $N_{12}(u(0))$ satisfies

$$
\phi_{12}(s)=\frac{m_{12}}{1-m_{22}} s+\tilde{c}_{12} s^{\tilde{\alpha}_{1}}+o\left(s^{\tilde{\alpha}_{1}}\right), \quad \text { as } s \downarrow 0 .
$$

On the other hand, we know that $m_{12} /\left(1-m_{22}\right)=a_{2} / a_{1}$.

We now consider case $d \geq 3$. We apply the operation $\tilde{\Pi}, d-2$ times, removing the types $d, d-1, \ldots, 3$ one after the other. We then obtain a two-type GW forest and we observe that the number of type 2 vertices that have only the root as type 1 ancestor is precisely the number of type 2 individuals that are trapped between two generations of $\Pi^{(1)}(F)$. Therefore, in view of the $d=2$ case above, it is not difficult to see that the Laplace exponent of $N_{12}(u(0))$ satisfies

$$
\phi_{12}(s)=\frac{a_{2}}{a_{1}} s+c_{12} s^{\underline{\alpha}}+o\left(s^{\underline{\alpha}}\right), \quad \text { as } s \downarrow 0,
$$

for some constant $c_{12}>0$. Finally, our claim follows by symmetry.

(ii) This is obtained by a similar induction argument. We only need to notice that for $i \in[d]$ and $j \in[d] \backslash\{i\}, \hat{N}_{i j}(n)=0$ when $x_{n}=i$.

2.2. Sub-exponential Bounds. The following lemma gives an exponential control on the height and number of components related to the $n$ first vertices in $d$-type GW forests. This extends Lemma 4 in Miermont (2008) which considers the finite variance case. Recall that for a forest $\mathbf{f} \in \mathbb{F}$, we let $1 \prec u_{\mathbf{f}}(0) \prec u_{\mathbf{f}}(1) \prec \cdots \prec$ $u_{\mathbf{f}}(\# \mathbf{f}-1)$ be the depth-first ordered list of its vertices. Recall also that $\Upsilon_{n}^{\mathbf{f}}$ is the index of the tree component to which $u_{\mathbf{f}}(n)$ belongs.

Lemma 2.4. There exist two constants $0<C_{1}, C_{2}<\infty$ (depending only on $\boldsymbol{\mu}$ ) such that for every $n \in \mathbb{N}, \mathbf{x} \in[d]^{\mathbb{N}}$ and $\eta>0$,

$$
\mathbf{P}^{\mathbf{x}}\left(\max _{0 \leq k \leq n}\left|u_{F}(k)\right| \geq n^{1-1 / \underline{\alpha}+\eta}\right) \leq C_{1}(n+1) \exp \left(-C_{2} n^{\eta}\right)
$$

and

$$
\mathbf{P}^{\mathbf{x}}\left(\Upsilon_{n}^{F} \geq n^{1-1 / \underline{\alpha}+\eta}\right) \leq C_{1} \exp \left(-C_{2} n^{\eta}\right)
$$

Proof: We observe that under $\mathbf{P}^{\mathbf{x}}$ and independently of $\mathbf{x}$, we have that

$$
\max _{0 \leq k \leq n}\left|u_{F}(k)\right| \leq \sum_{i \in[d]} \max _{0 \leq k \leq n}\left|u_{\Pi^{(i)}(F)}(k)\right| \quad \text { and } \quad \Upsilon_{n}^{F} \leq \sum_{i \in[d]} \Upsilon_{n}^{\Pi^{(i)}(F)}
$$

where each of the forests $\Pi^{(i)}(F)$, for $i \in[d]$, are critical non-degenerate monotype GW forests with offspring distribution in the domain of attraction of a stable law of index $\underline{\alpha} \in(1,2]$ by Proposition 2.1. Therefore, from the above inequalities, it is enough to prove the result only for the case $d=1$.

In this direction, let $\mu$ be a critical non-degenerate offspring distribution on $\mathbb{Z}_{+}$, with Laplace exponent given by

$$
\psi(s)=s+c s^{\alpha}+o\left(s^{\alpha}\right), \quad \text { as } s \downarrow 0,
$$

for $\alpha \in(1,2], s \in \mathbb{R}_{+}$and $c>0$ a constant. Let $\mathbf{P}$ be the law of a monotype $\mathrm{GW}$ forest with an infinite number of components and offspring distribution $\mu$. We then let $F$ be a monotype GW forest with law $\mathbf{P}$. 
It is well-known (Duquesne and Le Gall, 2002, Section 2.2) that $\left|u_{F}(k)\right|-1$ has the same distribution as the number of weak records for a random walk with step distribution $\mu(\{\cdot+1\})$ on $\{-1\} \cup \mathbb{Z}_{+}$, from time 1 up to time $k$. We denote by $\left(W_{n}, n \geq 0\right)$ such random walk and we also consider that is defined on some probability space $(\Omega, \mathcal{A}, \mathbb{P})$. By assumption, the step distribution of this random walk is centered and in the domain of attraction of stable law of index $\alpha \in(1,2]$. That is, $W_{n} / n^{1 / \alpha}$ converges in distribution towards a stable law of index $\alpha$ as $n \rightarrow \infty$. We fix $\tau_{0}=0$ and write $\tau_{j}, j \geq 0$, for the time of the $j$-th weak record of $\left(W_{n}, n \geq 0\right)$. Therefore, from Feller (1971) and Theorems 1 and 2 in Doney (1982), the sequence of random variables $\left(\tau_{j}-\tau_{j-1}, j \geq 1\right)$ is i.i.d. with Laplace exponent given by

$$
\tilde{\kappa}(\lambda)=-\log \mathbb{E}\left[\exp \left(-\lambda \tau_{1}\right)\right]=\tilde{C}_{1} \lambda^{1-1 / \alpha}+o\left(\lambda^{1-1 / \alpha}\right), \quad \text { as } \lambda \downarrow 0,
$$

for some constant $\tilde{C}_{1}>0$. We then bound the first probability by

$$
\mathbf{P}\left(\max _{0 \leq k \leq n}\left|u_{F}(k)\right| \geq n^{1-1 / \alpha+\eta}\right) \leq(n+1) \max _{0 \leq k \leq n} \mathbf{P}\left(\left|u_{F}(k)\right| \geq n^{1-1 / \alpha+\eta}\right) .
$$

Then, we notice that for $0 \leq k \leq n$ and $m \in \mathbb{N}$, we have that

$$
\begin{aligned}
\mathbf{P}\left(\left|u_{F}(k)\right|-1 \geq m\right) & =\mathbb{P}\left(\sum_{j=1}^{m}\left(\tau_{j}-\tau_{j-1}\right) \leq k\right) \\
& \leq e \mathbb{E}\left[\exp \left(-\sum_{j=1}^{m} \frac{\tau_{j}-\tau_{j-1}}{k}\right)\right] \\
& \leq \exp (1-m \tilde{\kappa}(1 / n)),
\end{aligned}
$$

where for the last inequality, we use the monotonicity of $\tilde{\kappa}$. Taking $m=$ $\left\lceil n^{1-1 / \alpha+\eta}\right\rceil-1$ and using (2.9), we get the first bound for large $n$ and thus for every $n$ up to tuning the constants $C_{1}, C_{2}$.

The proof for second bound is very similar. For $j \geq 1$, let $\# F_{j}$ be the number of vertices of the $j$-th tree component of the forest $F$. By the Otter-Dwass formula (see, e.g., Pitman, 2006, Chapter 5), under $\mathbf{P},\left(\# F_{i}, i \geq 1\right)$ is a sequence of i.i.d. random variables with common distribution

$$
\mathbf{P}\left(\# F_{1}=n\right)=n^{-1} \mathbb{P}\left(W_{n}=-1\right) .
$$

Using again the fact that the step distribution of $\left(W_{n}, n \geq 0\right)$ is centered and in the domain of attraction of a stable law of index $\alpha$, we obtain that

$$
\mathbf{P}\left(\# F_{1}=n\right)=\tilde{C}_{2} n^{-1-1 / \alpha}+o\left(n^{-1-1 / \alpha}\right), \quad \text { as } n \rightarrow \infty,
$$

where $\tilde{C}_{2}>0$ is some positive constant; see for example Lemma 1 in Kortchemski (2013). Therefore, an Abelian theorem (Feller, 1971, Theorem XIII.5.5) entails that the Laplace exponent $\kappa$ of the distribution of $\# F_{1}$, under $\mathbf{P}$, satisfies

$$
\kappa(\lambda)=\tilde{C}_{3} \lambda^{1-1 / \alpha}+o\left(\lambda^{1-1 / \alpha}\right), \quad \text { as } \lambda \downarrow 0,
$$

for some constant $\tilde{C}_{3}>0$. Noticing that $\left\{\Upsilon_{n}^{F}(n) \geq m\right\}=\left\{\sum_{i=1}^{m-1} \# F_{i} \leq n\right\}$, the second bound is then obtained analogously as the first one. Finally, we tune up the constants $C_{1}, C_{2}$ so that they match to both cases. 
2.3. Convergence of types. In order to compare the height process of the monotype GW forest $\Pi^{(i)}(F), i \in[d]$, with that of the $d$-type GW forest $F$, we must estimate the number of vertices of $F$ that stand between a type $i$ vertex of $\Pi^{(i)}(F)$ and one of its descendants. This is the purpose of the following result. Before that, we need some further notation.

Definition 2.5. We say that a sequence of positive numbers $\left(z_{n}, n \geq 0\right)$ is exponentially bounded if there are positive constants $c, C>0$ such that $z_{n} \leq C e^{-c n^{\epsilon}}$ for some $\varepsilon>0$ and large enough $n$. In order to simplify notations and avoid referring to the changing $\varepsilon$ 's and the constants $c$ and $C$, we write $z_{n}=\operatorname{oe}(n)$ in this case.

For a $d$-type forest $\mathbf{f} \in \mathbb{F}^{(d)}$ and a vertex $u \in \mathbf{f}$, we let $\operatorname{Anc}_{\mathbf{f}}^{u}(i)$ be the number of type $i$ ancestors of a vertex $u$. We provide the following key estimate for the height process which is the analogue of Proposition 5 in Miermont (2008).

Proposition 2.6. For every $\gamma>0$ and $\mathbf{x} \in[d]^{\mathbb{N}}$, we have that

$$
\max _{i \in[d]} \mathbf{P}^{\mathbf{x}}\left(\max _{0 \leq k \leq n}\left|H_{k}^{F}-\frac{\operatorname{Anc}_{F}^{u(k)}(i)}{a_{i} b_{i}}\right|>n^{1 / 2-1 / 2 \underline{\alpha}+\gamma}\right)=\operatorname{oe}(n) .
$$

On the other hand, observe that the height process of the monotype GW forest $\Pi^{(i)}(F)$ does not visit the vertices of type different from $i$, in words, it goes faster than the the height process of the $d$-type GW forest $F$. Then, in order to slow down the height process of $\Pi^{(i)}(F)$, we must adjust the time. We conclude this section with the following result which takes care of the number of vertices with type different from $i$ that stands between two consecutive type $i$ vertices in $\Pi^{(i)}(F)$. More precisely, for $\mathbf{f} \in \mathbb{F}^{(d)}$ and $n \geq 0$, we let

$$
\Lambda_{i}^{\mathbf{f}}(n)=\#\left\{0 \leq k \leq n: e_{\mathbf{f}}\left(u_{\mathbf{f}}(k)\right)=i\right\}
$$

be the number of type $i$ vertices standing before the $(n+1)$-th vertex in depth-first order. We let $u^{(i)}(0) \prec u^{(i)}(1) \prec \ldots$ be the type $i$ vertices of $\mathbf{f}$ arranged in depthfirst order, and we also consider the quantity $G_{i}^{\mathbf{f}}(n)=\#\left\{u \in \mathbf{f}: u \prec u^{(i)}(n)\right\}$, with the convention $G_{i}^{\mathbf{f}}\left(\# \mathbf{f}^{(i)}\right)=\# \mathbf{f}$. Similar notation holds if we consider trees instead of forests. Recall that $\mathbf{a}=\left(a_{1}, \ldots, a_{d}\right)$ is the left 1-eigenvector of the mean matrix M.

Proposition 2.7. For $i \in[d]$ and for any $\mathbf{x} \in[d]^{\mathbb{N}}$, under $\mathbf{P}^{\mathbf{x}}$, we have that

$$
\left(\frac{\Lambda_{i}^{F}(\lfloor n s\rfloor)}{n}, s \geq 0\right) \underset{n \rightarrow \infty}{\rightarrow}\left(a_{i} s, s \geq 0\right)
$$

in probability, for the topology of uniform convergence over compact subsets of $\mathbb{R}_{+}$.

Proof: We only need to prove that for $i \in[d], \varepsilon>0$ and for any $\mathbf{x} \in[d]^{\mathbb{N}}$, we have that

$$
\mathbf{P}^{\mathbf{x}}\left(\left|G_{i}^{F}(n)-a_{i}^{-1} n\right|>\varepsilon n\right)=0
$$

as $n \rightarrow \infty$. This will imply the convergence in probability for every rational number $s$ of $G_{i}^{F}(\lfloor n s\rfloor) n^{-1}$ towards $a_{i}^{-1} s$ as $n \rightarrow \infty$. Then, an application of Skorohod's representation theorem and a standard diagonal procedure entail that the above convergence holds for the uniform topology over compact subsets of $\mathbb{R}_{+}$. Finally, one notices that $\Lambda_{i}^{F}$ is the right-continuous inverse function of $G_{i}^{F}$ which leads to our statement. 
In this direction, for $\mathbf{f} \in \mathbb{F}^{(d)}$, we recall that $\Pi^{(i)}(\mathbf{f})$ denotes the monotype forest obtained after applying the projection function described in Section 2.1. Recall that for $k \geq 0$ and $j \in[d] \backslash\{i\}, N_{i j}(k):=N_{i j}\left(u^{(i)}(k)\right)$ denotes the number of type $j$ vertices that have been deleted between $u^{(i)}(k)$ and its children during the operation $\Pi^{(i)}$. Similarly, we define the quantity $N_{i j}^{\prime}(k)$ which counts only the type $j$ vertices that come before $u^{(i)}(n)$ in depth-first order. Since $\sum_{j \neq i} a_{j} / a_{i}=1-1 / a_{i}$, we notice that

$$
G_{i}^{\mathbf{f}}(n)-a_{i}^{-1} n=\sum_{j \neq i}\left(R_{1}^{\mathbf{f}}(j ; n)+R_{2}^{\mathbf{f}}(j ; n)+R_{3}^{\mathbf{f}}(j ; n)\right)
$$

for $n \geq 0$ and where for $j \in[d] \backslash\{i\}$,

$$
R_{1}^{\mathbf{f}}(j ; n)=\sum_{k=0}^{n-1}\left(N_{i j}^{\prime}(k)-N_{i j}(k)\right) \mathbb{1}_{\left\{u^{(i)}(k) \vdash u^{(i)}(n)\right\}}, \quad R_{2}^{\mathbf{f}}(j ; n)=\sum_{k=1}^{\Upsilon_{n}^{\mathbf{f}}} \hat{N}_{i j}(k),
$$

and

$$
R_{3}^{\mathbf{f}}(j ; n)=\sum_{k=0}^{n-1}\left(N_{i j}(k)-a_{j} / a_{i}\right) .
$$

We next estimate the probability that these tree terms are large, when we consider a $d$-type GW forest. We fix $\varepsilon>0,0<\delta<1 / \underline{\alpha}$ and write $z_{n}=n^{1-1 / \underline{\alpha}+\delta}$. We observe that

$$
\left|R_{1}^{\mathbf{f}}(j ; n)\right| \leq \sum_{k=0}^{n-1} N_{i j}(k) \mathbb{1}_{\left\{u^{(i)}(k) \vdash u^{(i)}(n)\right\}}
$$

and

$$
\#\left\{k \geq 0: u^{(i)}(k) \vdash u^{(i)}(n)\right\} \leq \operatorname{Anc}_{\mathbf{f}}^{u^{(i)}(n)}(i) \leq \max _{0 \leq k \leq n} H_{k}^{\Pi^{(i)}(F)} .
$$

Thus, according to our estimate for the height of GW forests in Lemma 2.4, we get that

$$
\mathbf{P}^{\mathbf{x}}\left(\left|R_{1}^{F}(j ; n)\right|>\varepsilon n^{1+\delta}\right) \leq \mathbf{P}^{\mathbf{x}}\left(\sum_{k=0}^{\left\lfloor z_{n}\right\rfloor} N_{i j}(k)>\varepsilon n^{1+\delta}\right)+\operatorname{oe}(n) .
$$

Moreover, for every $\beta \in(0,1 / 2)$,

$$
\begin{aligned}
& \mathbf{P}^{\mathbf{x}}\left(\left|R_{1}^{F}(j ; n)\right|>\varepsilon n^{1+\delta}\right) \\
& \leq \mathbf{P}^{\mathbf{x}}\left(\left\{\sum_{k=1}^{\left\lfloor z_{n}\right\rfloor} N_{i j}(k)>\varepsilon n^{1+\delta}\right\} \cap\left\{\forall k \in\left\{0,1, \ldots,\left\lfloor z_{n}\right\rfloor\right\}: N_{i j}(k)<(1-\beta) \varepsilon n^{1+\delta}\right\}\right) \\
& +\mathbf{P}^{\mathbf{x}}\left(\max _{1 \leq k \leq\left\lfloor z_{n}\right\rfloor} N_{i j}(k)>(1-\beta) \varepsilon n^{1+\delta}\right)+\operatorname{oe}(n) .
\end{aligned}
$$

We recall that under $\mathbf{P}^{\mathbf{x}}$, the random variables $\left(N_{i j}(k), k \geq 0\right)$ are i.i.d. with law in the domain of attraction of a stable law of index $\underline{\alpha} \in(1,2]$ by Proposition 2.3 (i). Then,

$$
\mathbf{P}^{\mathbf{x}}\left(\max _{0 \leq k \leq\left\lfloor z_{n}\right\rfloor} N_{i j}(k)>(1-\beta) \varepsilon n^{1+\delta}\right)=1-\left(1-\mathbf{P}^{\mathbf{x}}\left(N_{i j}(0)>(1-\beta) \varepsilon n^{1+\delta}\right)\right)^{\left\lfloor z_{n}\right\rfloor}
$$


which tends to 0 as $n \rightarrow \infty$. On the other hand, the first term in the right-hand side of (2.13) also tends to 0 as $n \rightarrow \infty$. To see this, note that the event in the first term may hold only if there are two distinct values of $k \in\left\{0,1, \ldots,\left\lfloor z_{n}\right\rfloor\right\}$ such that $N_{i j}(k) \geq \beta \varepsilon n /\left\lfloor z_{n}\right\rfloor$. We thus conclude that

$$
\mathbf{P}^{\mathbf{x}}\left(\left|R_{1}^{F}(j ; n)\right|>\varepsilon n^{1+\delta}\right) \underset{n \rightarrow \infty}{\rightarrow} 0 .
$$

Following exactly the same argument, using the bound in Lemma 2.4 on the number of components of $d$-type GW forests and Proposition 2.3 (ii), we obtain that

$$
\mathbf{P}^{\mathbf{x}}\left(\left|R_{2}^{F}(j ; n)\right|>\varepsilon n^{1+\delta}\right) \underset{n \rightarrow \infty}{\rightarrow} 0 .
$$

Finally, the estimate

$$
\mathbf{P}^{\mathbf{x}}\left(\left|R_{3}^{F}(j ; n)\right|>\varepsilon n^{1+\delta}\right) \underset{n \rightarrow \infty}{\rightarrow} 0,
$$

follows by the law of large numbers, since Proposition 2.3 (i) entails that the mean of $N_{i j}(0)$ is $a_{j} / a_{i}$.

Therefore, the estimates (2.14), (2.15) and (2.16), when combined with (2.12) imply the convergence (2.11).

\section{Proof of Theorem 1.2 and 1.3}

In this section, we prove our main results.

Proof of Theorem 1.2: We observe that for $n \geq 0$ and any $s \geq 0$, we have

$$
\begin{aligned}
& \left|H_{\lfloor n s\rfloor}^{F}-\frac{H_{\Lambda_{i}^{F}(\lfloor n s\rfloor)-1}^{\Pi^{(i)}(F)}}{a_{i} b_{i}}\right| \\
& \leq\left|H_{\lfloor n s\rfloor}^{F}-\frac{\operatorname{Anc}_{F}^{u(\lfloor n s\rfloor)}(i)}{a_{i} b_{i}}\right|+\frac{1}{a_{i} b_{i}}\left|H_{\Lambda_{i}^{F}(\lfloor n s\rfloor)-1}^{\Pi^{(i)}(F)}-\operatorname{Anc}_{F}^{u(\lfloor n s\rfloor)}(i)\right| .
\end{aligned}
$$

By Proposition 2.6, under $\mathbf{P}^{\mathbf{x}}$, the first term on the right hand side tends to 0 in probability as $n \rightarrow \infty$, uniformly over compact subsets of $\mathbb{R}_{+}$. On the other hand, from equation (15) in Miermont (2008), we get that

$$
\left|H_{\Lambda_{i}^{F}(\lfloor n s\rfloor)-1}^{\Pi^{(i)}(F)}-\operatorname{Anc}_{F}^{u(\lfloor n s\rfloor)}(i)\right| \leq\left|H_{\Lambda_{i}^{F}(\lfloor n s\rfloor)-1}^{\Pi^{(i)}(F)}-H_{\Lambda_{i}^{F}(\lfloor n s\rfloor)}^{\Pi^{(i)}(F)}\right|+1 .
$$

Recall that under $\mathbf{P}^{\mathbf{x}}, \Pi^{(i)}(F)$ is a critical non-degenerate monotype GW forest in the domain of attraction of a stable law of index $\underline{\alpha} \in(1,2]$ by Proposition 2.1. Then, Theorem 2.3.2 in Duquesne and Le Gall (2002) implies that

$$
\frac{1}{n^{1-1 / \underline{\alpha}}} \max _{0 \leq k \leq n}\left|H_{k-1}^{\Pi^{(i)}(F)}-H_{k}^{\Pi^{(i)}(F)}\right| \underset{n \rightarrow \infty}{\rightarrow} 0
$$

in probability, under $\mathbf{P}^{\mathbf{x}}$, and it follows that

$$
\left(\frac{1}{n^{1-1 / \underline{\alpha}}}\left(H_{\lfloor n s\rfloor}^{F}-\frac{1}{a_{i} b_{i}} H_{\Lambda_{i}^{F}(\lfloor n s\rfloor)}^{\Pi^{(i)}(F)}\right), s \geq 0\right) \underset{n \rightarrow \infty}{\rightarrow} 0
$$

in probability for the topology of uniform convergence over compact sets of $\mathbb{R}_{+}$. Finally, Proposition 2.7 and Theorem 2.3.2 in Duquesne and Le Gall (2002) imply that

$$
\left(\frac{1}{n^{1-1 / \underline{\alpha}}} H_{\Lambda_{i}^{F}(\lfloor n s\rfloor)}^{\Pi^{(i)}(F)}, s \geq 0\right) \underset{n \rightarrow \infty}{\stackrel{d}{\longrightarrow}}\left(\frac{a_{i}^{1 / \underline{\alpha}} b_{i}}{\bar{c}} H_{a_{i} s}, s \geq 0\right) .
$$


Moreover, we deduce from the scaling property of the height process $H$ that $\left(H_{a_{i} s}, s \geq 0\right) \stackrel{d}{=}\left(a_{i}^{1-1 / \underline{\alpha}} H_{s}, s \geq 0\right)$; see, e.g., Section 3.1 in Duquesne and Le Gall (2002). Therefore, the result in Theorem 1.2 follows now from (3.1).

Let us now prove Theorem 1.3.

Proof of Theorem 1.3: For $n \geq 0, i \in[d]$ and any $s \geq 0$, we recall that $\Lambda_{i}^{F}(\lfloor n s\rfloor)$ denotes the number of type $i$ individuals standing before the $(\lfloor n s\rfloor+1)$-th individual in depth-first order which we called $u(\lfloor n s\rfloor)$. Since all the roots of the forest $F$ have type $i$, we claim that

$$
\Upsilon_{\Lambda_{i}^{F}(\lfloor n s\rfloor)}^{\Pi^{(i)}(F)}=\Upsilon_{\lfloor n s\rfloor} .
$$

To see this, we observe that $u(\lfloor n s\rfloor)$ and the last vertex of type $i$ before $u(\lfloor n s\rfloor)$ in depth-first order belong to the same tree component. Therefore, the label of the tree component of $F$ containing $u(\lfloor n s\rfloor)$ is the same as the label of the tree component of $\Pi^{(i)}(F)$ containing the $\Lambda_{i}^{F}(\lfloor n s\rfloor)$-th vertex.

Let $W^{\Pi^{(i)}(F)}=\left(W_{n}^{\Pi^{(i)}(F)}, n \geq 1\right)$ be the Lukasiewicz path associated with monotype GW forest $\Pi^{(i)}(F)$ (see proof of Lemma 2.4 for the definition) which according to Proposition 1.2 has offspring distribution that belongs to the domain of attraction of a stable law of index $\underline{\alpha} \in(1,2]$. We need the following property of Lukasiewicz path,

$$
\inf _{0 \leq k \leq n} W_{k}^{\Pi^{(i)}(F)}=-\Upsilon_{n}^{\Pi^{(i)}(F)},
$$

for $n \geq 1$; see for example Duquesne (2003). The result now follows from Corollary 2.5.1 in Duquesne (2003) and similar arguments as at the end of proof of Theorem 1.2.

\section{Applications}

4.1. Maximal height of multitype $G W$ trees. In this section, we present a natural consequence of Theorems 1.2 and 1.3 which generalizes the result of Miermont (2008) on the maximal height in the finite covariance case. For a tree $\mathbf{t} \in \mathbb{T}$, we let ht $(\mathbf{t})$ be the maximal height of a vertex in $\mathbf{t}$. Recall that $I_{s}$ is the infimum at time $s$ of the strictly stable spectrally positive Lévy process $Y(\underline{\alpha})$.

Corollary 4.1. For $i \in[d]$, let $T$ be a d-type $G W$ tree distributed according to $\mathbf{P}^{(i)}$ whose offspring distribution satisfies the main assumptions. Then,

$$
\lim _{n \rightarrow \infty} n \mathbf{P}^{(i)}(h t(T) \geq n)=b_{i}(\underline{\alpha}-1)((\underline{\alpha}-1) \bar{c})^{\frac{\underline{\alpha}}{1-\underline{\alpha}}} .
$$

Proof: The proof of this assertion is very similar of Corollary 1 in Miermont (2008). The only difference that we are now considering that the rescaled height process of multitype GW forest converges to height process associated with the strictly stable spectrally positive Lévy process $Y^{(\underline{\alpha})}$. Let $F$ be a $d$-type GW forest distributed according to $\mathbf{P}^{(\mathbf{i})}$ whose offspring distribution satisfies the main assumptions. For $k \geq 1$, we denote by $\tau_{k}$ the first hitting time of $k$ by $\left(\Upsilon_{n}^{F}, n \geq 0\right)$ and for $x \geq 0$, we 
write $\varrho_{x}$ for the first hitting time of $x$ by $-I=\left(-I_{s}, s \geq 0\right)$. From Theorem 1.2 and 1.3 , we have that

$$
\left(\frac{1}{n} H_{n^{\frac{\alpha}{\alpha-1}} s}^{F}, 0 \leq s \leq \tau_{n}\right) \underset{n \rightarrow \infty}{\stackrel{d}{\longrightarrow}}\left(\frac{1}{\bar{c}} H_{s}, 0 \leq s \leq \varrho_{b_{i} \bar{c}^{-1}}\right),
$$

under $\mathbf{P}^{(\mathbf{i})}$. Let $\left(F_{k}, k \geq 1\right)$ be the tree components of the multitype GW forest $F$. Then, the above convergence implies that

$$
\begin{aligned}
\lim _{n \rightarrow \infty} \mathbf{P}^{(\mathbf{i})}\left(\max _{1 \leq k \leq n} h t\left(F_{k}\right)<n\right) & =\mathbf{P}\left(H_{s} \leq \bar{c}, \text { for all } 0 \leq s \leq \varrho_{b_{i} \bar{c}^{-1}}\right) \\
& =\exp \left(-\frac{b_{i}}{\overline{c_{i}}} N\left(\frac{1}{\bar{c}} \sup H \geq 1\right)\right) \\
& =\exp \left(-b_{i}(\underline{\alpha}-1)((\underline{\alpha}-1) \bar{c})^{\frac{\alpha}{1-\underline{\alpha}}}\right),
\end{aligned}
$$

where $N$ is the Itô excursion measure of $Y^{(\underline{\alpha})}$ above its infimum (see e.g. Chapter VIII.2 in Bertoin (1996) for details), and where we have used the Corollary 1.4.2 in Duquesne and Le Gall (2002) for the equality. Recall that under $\mathbf{P}^{(\mathbf{i})}$, the tree components $\left(F_{k}, k \geq 1\right)$ are independent multitype GW trees. Therefore, the identity

$$
\mathbf{P}^{(\mathbf{i})}\left(\max _{1 \leq k \leq n} \operatorname{ht}\left(F_{k}\right)<n\right)=\left(1-\mathbf{P}^{(i)}(\mathrm{ht}(T) \geq n)\right)^{n} .
$$

yields our claim.

4.2. Alternating two-type $G W$ tree. We consider a particular family of multitype GW trees known as alternating two-type GW trees, in which vertices of type 1 only give birth to vertices of type 2 and vice versa. More precisely, given two probability measures $\mu_{2}^{(1)}$ and $\mu_{1}^{(2)}$ on $\mathbb{Z}_{+}$, we consider a two-type GW tree where every vertex of type 1 (resp. type 2 ) has a number of type 2 (resp. type 1 ) children distributed according to $\mu_{2}^{(1)}$ (resp. $\mu_{1}^{(2)}$ ), all independent of each other. We denote by $\boldsymbol{\mu}_{\text {alt }}$ the offspring distribution on $\mathbb{Z}_{+}^{2}$ of this particular two-type GW tree. We let

$$
m_{1}=\sum_{z \in \mathbb{Z}_{+}} z \mu_{2}^{(1)}(\{z\}) \quad \text { and } \quad m_{2}=\sum_{z \in \mathbb{Z}_{+}} z \mu_{1}^{(2)}(\{z\})
$$

be the means of the measures $\mu_{2}^{(1)}$ and $\mu_{1}^{(2)}$, respectively. We make the assumption that $\mu_{2}^{(1)}(\{1\})+\mu_{1}^{(2)}(\{1\})<2$ to discard degenerate cases, and also exclude the trivial case $m_{1} m_{2}=0$. We observe that the mean matrix associated with $\boldsymbol{\mu}_{\text {alt }}$ is irreducible and it admits $\rho=m_{1} m_{2}$ as a unique positive eigenvalue. We then say that $\boldsymbol{\mu}_{\text {alt }}$ is sub-critical if $m_{1} m_{2}<1$, critical if $m_{1} m_{2}=1$ and supercritical if $m_{1} m_{2}>1$. In the sequel, we assume that offspring distribution is also critical. We observe then that the normalized left and right 1-eigenvectors are given by

$$
\mathbf{a}=\left(a_{1}, a_{2}\right)=\left(\frac{1}{1+m_{1}}, \frac{1}{1+m_{2}}\right), \quad \text { and } \quad \mathbf{b}=\left(b_{1}, b_{2}\right)=\left(\frac{1+m_{1}}{2}, \frac{1+m_{2}}{2}\right) .
$$

Following the notation of Section 1.3, we denote by $\mathbf{P}_{\text {alt }}^{(i)}$ the law of a two-type GW tree with offspring distribution $\boldsymbol{\mu}_{\text {alt }}$ and root type $i \in[2]$, i.e., it is the law of an alternating two-type $\mathrm{GW}$ tree with root type $i$. We make the next extra assumptions on the offspring distribution: 
$\left(\mathbf{H}_{1}^{\prime}\right) \mu_{2}^{(1)}$ is a geometric distribution, i.e. there exists $p \in(0,1)$ such that

$$
\mu_{2}^{(1)}(\{z\})=(1-p) p^{z}, \quad z \in \mathbb{Z}_{+} .
$$

We observe that its Laplace exponent satisfies

$$
\psi_{1}(s)=\frac{p}{1-p} s+\frac{1}{2} \frac{p}{(1-p)^{2}} s^{2}+o\left(s^{2}\right), \quad s \downarrow 0,
$$

for $s \in \mathbb{R}_{+}$. In particular, $m_{1}=p /(1-p)$.

$\left(\mathbf{H}_{2}^{\prime}\right) \mu_{1}^{(2)}$ is in the domain of attraction of a stable law of index $\alpha \in(1,2]$, that is, its Laplace exponent satisfies

$$
\psi_{2}(s)=m_{2} s+s^{\alpha} L(s)+o\left(s^{\alpha}\right), \quad s \downarrow 0,
$$

for $s \in \mathbb{R}_{+}$and where $L: \mathbb{R}_{+} \rightarrow \mathbb{R}_{+}$is a slowly varying function at zero.

The following result is a conditioned version of Theorem 1.2 for this particular two-type GW tree. More precisely, we show that after a proper rescaling the height process of a critical alternating two-type GW tree whose offspring distribution satisfies $\left(\mathbf{H}_{1}^{\prime}\right)$ and $\left(\mathbf{H}_{2}^{\prime}\right)$ converges to the normalized excursion of the continuoustime height process associated with a strictly stable spectrally positive Lévy process with index $\alpha$. We stress that the improvement of the convergence in Theorem 1.2 is because we are able to establish a conditioned version of Proposition 2.7 for this very particular GW tree. This allows us to adapt the proof of Theorem 2 in Miermont (2008), in the case where only the geometric part of the offspring distribution does have small exponential moments.

Before providing a rigorous statement, we need to introduce some further notation. We consider a function $\bar{L}: \mathbb{R}_{+} \rightarrow \mathbb{R}_{+}$given by

$$
\bar{L}(s)=\left(\frac{1}{2} \frac{p}{(1-p)^{2}} a_{1} b_{2}^{2} \mathbb{1}_{\{\alpha=2\}}+a_{2} b_{1}^{\alpha} L(s)\right), \quad \text { for } s \in \mathbb{R}_{+},
$$

which is a slowly varying function at zero. We write $\tilde{L}: \mathbb{R}_{+} \rightarrow \mathbb{R}_{+}$for a slowly varying function at infinity that satisfies

$$
\lim _{s \rightarrow \infty}\left(\frac{1}{\tilde{L}(s)}\right)^{\alpha} \bar{L}\left(\frac{1}{s^{1 / \alpha} \tilde{L}(s)}\right)=1,
$$

This function is known in the literature as the conjugate of $\bar{L}$. The existence of such a function is due to a result of de Bruijn; for a proof of this fact and more information about conjugate functions, see Section 1.5.7 in Bingham et al. (1989). In what follows, we let $\left(B_{n}, n \geq 1\right)$ be a sequence positive integers such that $B_{n}=\tilde{L}(n) n^{1 / \alpha}$.

Finally, recall from the beginning of Section 1.4 that $H^{\mathbf{t}}=\left(H_{n}^{\mathbf{t}}, n \geq 0\right)$ denotes the height process of the tree $\mathbf{t} \in \mathbb{T}$.

Theorem 4.2. Let $T$ be an alternating two-type $G W$ tree distributed according to $\mathbf{P}_{\text {alt }}^{(1)}$. Then for $j=1,2$, under the law $\mathbf{P}_{\text {alt }}^{(1)}\left(\cdot \mid \# T^{(j)}=n\right)$, the following convergence in distribution holds on $\mathbb{D}([0,1], \mathbb{R})$ :

$$
\left(\frac{B_{n}}{n} H_{\lfloor \# T s\rfloor}^{T}, 0 \leq s \leq 1\right) \underset{n \rightarrow \infty}{\stackrel{d}{\longrightarrow}}\left(a_{j}^{1 / \alpha-1} H_{s}^{e x c}, 0 \leq s \leq 1\right),
$$

where $H^{\text {exc }}$ is the normalized excursion of the continuous-time height process process associated with a strictly stable spectrally positive Lévy process $Y^{(\alpha)}=\left(Y_{s}, s \geq 0\right)$ of index $\alpha$ and with Laplace exponent $\mathbb{E}\left(\exp \left(-\lambda Y_{s}\right)\right)=\exp \left(-s \lambda^{\alpha}\right)$, for $\lambda \in \mathbb{R}_{+}$. 
In recent years, this special family of two-type GW trees has been the subject of many studies due to their remarkable relationship with the study of several important objects and models of growing relevance in modern probability such that random planar maps (Le Gall and Miermont, 2011), percolation on random maps (Curien and Kortchemski, 2015), non-crossing partitions (Kortchemski and Marzouk, 2017), to mention just a few. On the other hand, up to our knowledge the result of Theorem 4.2 has not been proved before under our assumptions on the offspring distribution. Therefore, we believe that this may open the way to investigate new aspects related to the models mentioned before.

The proof of Theorem 4.2 relies on some intermediate results. We let $T$ be a two-type GW tree with law $\mathbf{P}_{\text {alt }}^{(1)}$. We first characterize the law of the reduced forest $\Pi^{(j)}(T)$, for $j=1,2$.

Corollary 4.3. For $j=1,2$, under the law $\mathbf{P}_{\text {alt }}^{(1)}$, the tree $\Pi^{(j)}(T)$ is a critical monotype $G W$ forest with non-degenerate offspring distribution $\bar{\mu}_{j}$ in the domain of attraction of a stable law of index $\alpha$, i.e., its Laplace exponent satisfies that

$$
\bar{\psi}_{j}(s)=s+\frac{1}{a_{j}}\left(\frac{s}{b_{j}}\right)^{\alpha} \bar{L}(s)+o\left(s^{\alpha}\right), \quad s \downarrow 0 .
$$

for $s \in \mathbb{R}_{+}$and where the function $\bar{L}$ is defined in (4.1).

Proof: The results follows from Lemma 2.2, after some simple computations.

The next step in order to pass from unconditional statements to conditional ones is the following estimate for the number of vertices of some specific type in multitype GW trees.

Lemma 4.4. Let $T$ be a d-type $G W$ tree distributed according to $\mathbf{P}^{(i)}$, for $i \in[d]$. Then, for every $j \in[d]$ :

(i) For some constant $C_{i j}>0$, we have that

$$
\mathbf{P}^{(i)}\left(\# T^{(j)}=n\right)=C_{i j} n^{-1-1 / \underline{\alpha}}+o\left(n^{-1-1 / \underline{\alpha}}\right), \quad \text { as } n \rightarrow \infty,
$$

where it is understood that the limit is taken along values for which the probability on the left-hand side is strictly positive.

(ii) The laws of the number of tree components of $\Pi^{(j)}(T)$, under $\mathbf{P}^{(i)}\left(\cdot \mid \# T^{(j)}=\right.$ $n$ ), converge weakly as $n \rightarrow \infty$.

Proof: This very similar to Lemma 6 and Lemma 7 in Miermont (2008) and the proof is carried out with mild modifications.

Finally, the last ingredient is a conditioned version of Proposition 2.7 for the alternating two-type GW tree.

Proposition 4.5. For $j=1,2$, under $\mathbf{P}_{\text {alt }}^{(1)}\left(\cdot \mid \# T^{(j)}=n\right)$, we have that

$$
\left(\frac{\Lambda_{j}^{T}(\lfloor \# T s\rfloor)}{n}, 0 \leq s \leq 1\right) \underset{n \rightarrow \infty}{\rightarrow}(s, 0 \leq s \leq 1)
$$

in probability. 
Proof: We prove the statement only when $j=1$. The case $j=2$ follows by making occasional changes in the proof below, observing that

$$
\Lambda_{1}^{T}(\# T)+\Lambda_{2}^{T}(\# T)=\# T^{(1)}+\# T^{(2)}=\# T .
$$

We based our proof on a bijection $\mathcal{G}$ due to Janson and Stefánsson (2015) which maps the alternating two-type GW tree to a standard monotype GW tree. More precisely, the tree $\mathcal{G}(T)$ has the same vertices as $T$, but edges are different and are defined as follows. For every type 1 vertex $u$ we repeat the following operation: let $u_{0}$ be the parent of $u$ (if $u \neq \varnothing$ ) and we list the children of $u$ in lexicographical order $u_{1} \prec u_{2} \prec \cdots \prec u_{k}$. If $u \neq \varnothing$ draw the edge between $u_{0}$ and $u_{1}$ and then edges between $u_{1}$ and $u_{2}, \ldots, u_{k-1}$ and $u_{k}$ and finally between $u_{k}$ and $u$. If $u$ is a type 1 vertex and a leaf this reduces to draw the edge between $u_{0}$ and $u$. One can check that $\mathcal{G}(T)$ defined by this procedure is a tree and rooted at the corner between the root of $T$ and its first child. Roughly speaking, this mapping has the property that every vertex of type 1 is mapped to a leaf, and every type 2 vertex with $k \geq 0$ children is mapped to a vertex with $k+1$ children (the interest reader is referred to Section 3 in Janson and Stefánsson (2015), for details). Moreover, Janson and Stefánsson showed that under $\mathbf{P}_{\text {alt }}^{(1)}, \mathcal{G}(T)$ is a monotype GW tree with offspring distribution given by

$$
\nu(\{0\})=1-p, \quad \text { and } \quad \nu(\{z\})=p \mu_{2}(\{z-1\}), \quad \text { for } z \in \mathbb{N} .
$$

Moreover, the criticality assumption $m_{1} m_{2}=1$ implies that the offspring $\nu$ is critical, i.e. it has mean 1. We also notice that $\Lambda_{1}^{T}(\# T)=\# T^{(1)}$ is exactly the number of leaves of the monotype $\mathrm{GW}$ tree $\mathcal{G}(T)$. Then, Lemma 2.5 in Kortchemski (2012) which is a law of large numbers for the number of leaves of monotype GW trees, implies that for every $\varepsilon>0$,

$$
\mathbf{P}_{\text {alt }}^{(1)}\left(\sup _{0 \leq s \leq 1}\left|\frac{\Omega^{\mathcal{G}(T)}(\lfloor \# T s\rfloor)}{\# T s}-(1-p)\right|>\varepsilon \mid \# T \geq n\right)=\mathrm{oe}(n),
$$

where $\Omega^{\mathcal{G}(T)}(n)$ denotes the number of leaves standing before the $(n+1)$-th vertex in depth-first order in the tree $\mathcal{G}(T)$. We observe that the left 1-eigenvector $a_{1}=1-p$. By Lemma 4.4, we deduce that

$$
\mathbf{P}_{\text {alt }}^{(1)}\left(\sup _{0 \leq s \leq 1}\left|\frac{\Omega^{\mathcal{G}(T)}(\lfloor \# T s\rfloor)}{\# T s}-a_{1}\right|>\varepsilon \mid \# T^{(1)}=n\right)=\operatorname{oe}(n) .
$$

Then, if we admit for a while that

$$
\mathbf{P}_{\text {alt }}^{(1)}\left(\left|\frac{\# T}{n}-\frac{1}{a_{1}}\right|>\varepsilon \mid \# T^{(1)}=n\right)=\text { oe }(n) .
$$

We conclude that under $\mathbf{P}_{\text {alt }}^{(1)}\left(\cdot \mid \# T^{(1)}=n\right)$, we have that

$$
\left(\frac{\Omega^{\mathcal{G}(T)}(\lfloor \# T s\rfloor)}{n}, 0 \leq s \leq 1\right) \underset{n \rightarrow \infty}{\rightarrow}(s, 0 \leq s \leq 1),
$$

in probability, and the result follows by noticing that

$$
\left|\Lambda_{1}^{T}(\lfloor \# T s\rfloor)-\Omega^{\mathcal{G}(T)}(\lfloor \# T s\rfloor)\right| \leq c_{\mathcal{G}(T)}(\lfloor \# T s\rfloor)
$$

(recall that $c_{\mathbf{t}}(u)$ denotes the number of children of the vertex $u$ in the tree t) where the term in the right hand of the inequality is $o(n)$ uniformly in $s \in[0,1]$, in probability, by Theorem 2 in Kortchemski (2017). 
Let us now turn to the proof of (4.3). First, we observe that for $0<\varepsilon<a_{1}^{-1}$, we have that

$$
\begin{aligned}
\mathbf{P}_{\text {alt }}^{(1)}\left(\left|\frac{\# T}{n}-\frac{1}{a_{1}}\right|>\varepsilon, \# T^{(1)}=n\right) & =\mathbf{P}_{\text {alt }}^{(1)}\left(\# T>\left(\frac{1}{a_{1}}+\varepsilon\right) n, \# T^{(1)}=n\right) \\
& +\mathbf{P}_{\text {alt }}^{(1)}\left(\# T<\left(\frac{1}{a_{1}}-\varepsilon\right) n, \# T^{(1)}=n\right) .
\end{aligned}
$$

The idea is to show that the two term on the right-hand side are oe $(n)$. We start with the first term. We notice that

$$
\begin{aligned}
& \mathbf{P}_{\text {alt }}^{(1)}\left(\# T>\left(\frac{1}{a_{1}}+\varepsilon\right) n, \# T^{(1)}=n\right) \\
& \leq \sum_{k=n}^{\infty} \mathbf{P}_{\text {alt }}^{(1)}\left(\# T=k, \# T^{(1)}<\left(\frac{1}{a_{1}}+\varepsilon\right)^{-1} n\right)
\end{aligned}
$$

By recalling that $\# T^{(1)}$ is the number of leaves of the monotype $\mathrm{GW}$ tree $\mathcal{G}(T)$, Lemma 2.7 (ii) in Kortchemski (2012) implies that terms in the sum are oe $(n)$. This entails that the first term on the right-hand side of (4.4) is oe $(n)$. We now focus on the second term. We write

$$
\begin{aligned}
\mathbf{P}_{\text {alt }}^{(1)}\left(\# T>\left(\frac{1}{a_{1}}+\varepsilon\right) n, \# T^{(1)}=n\right) \\
\quad \leq \sum_{k=n}^{\left\lfloor\left(a_{1}^{-1}-\varepsilon\right) n\right\rfloor} \mathbf{P}_{\text {alt }}^{(1)}\left(\# T=k, \# T^{(1)}>\left(\frac{1}{a_{1}}-\varepsilon\right)^{-1} n\right)
\end{aligned}
$$

By using Proposition 1.6 in Kortchemski (2012), we get that

$$
\begin{aligned}
\mathbf{P}_{\mathrm{alt}}^{(1)} & \left(\# T>\left(\frac{1}{a_{1}}+\varepsilon\right) n, \# T^{(1)}=n\right) \\
& \leq \sum_{k=n}^{\left\lfloor\left(a_{1}^{-1}-\varepsilon\right) n\right\rfloor} \frac{1}{n} \mathbf{P}_{\mathrm{alt}}^{(1)}\left(\frac{1}{r} \sum_{r=1}^{k} \mathbf{1}_{\left\{X_{r}=-1\right\}}>\left(\frac{1}{a_{1}}-\varepsilon\right)^{-1}\right),
\end{aligned}
$$

where $\left(X_{r}, r \geq 1\right)$ is a sequence of i.i.d. random variables with common distribution $\nu(\{\cdot+1\})$ on $\{-1\} \cup \mathbb{Z}_{+}$. Then, an application of Lemma 2.2 (i) in Kortchemski (2012) shows that this is oe $(n)$. Therefore, we have proved that

$$
\mathbf{P}_{\text {alt }}^{(1)}\left(\left|\frac{\# T}{n}-\frac{1}{a_{1}}\right|>\varepsilon, \# T^{(1)}=n\right)=\mathrm{oe}(n) .
$$

Finally, an appeal to Lemma 4.4 (i) completes the proof of (4.3).

We have now all the ingredients to give the proof of Theorem 4.2.

Proof of Theorem 4.2: Recall from Corollary 4.3 that $\Pi^{(j)}(T)$ under $\mathbf{P}_{\text {alt }}^{(1)}$ is a nondegenerate, critical GW forest with offspring distribution $\bar{\mu}_{j}$ in the domain of attraction of a stable law of index $\alpha \in(1,2]$. Thus, by first conditioning on the number of tree components, we obtain using Lemma 4.4 (ii) and Theorem 3.1 Duquesne (2003) that under $\mathbf{P}_{\text {alt }}^{(1)}\left(\cdot \mid \# T^{(j)}=n\right)$,

$$
\left(\frac{B_{n}}{n} H_{\lfloor n s\rfloor}^{\Pi^{(j)}(T)}, 0 \leq s \leq 1\right) \underset{n \rightarrow \infty}{\stackrel{d}{\longrightarrow}}\left(a_{j}^{1 / \alpha} b_{j} H_{s}^{\mathrm{exc}}, 0 \leq s \leq 1\right),
$$


where the convergence is in distribution on $\mathbb{D}([0,1], \mathbb{R})$. To see this, we observe that conditional on the number of tree components to be $r$, the GW forest $\Pi^{(j)}(T)$ is composed of $r$ independent GW trees with the same offspring distribution $\bar{\mu}_{j}$. On the other hand, conditioning the sum of their size to be $n$, only one of these trees has size of order $n$, while the other $r-1$ trees have total size $o(n)$ with high probability. This implies that the latter do not contribute to the limit. We refer to Theorem 5.4 in Kortchemski and Marzouk (2016) for details. Then, from Proposition 4.5, we obtain that under $\mathbf{P}_{\text {alt }}^{(1)}\left(\cdot \mid \# T^{(j)}=n\right)$,

$$
\left(\frac{B_{n}}{n} H_{\Lambda_{j}^{T}(\lfloor \# T s\rfloor)}^{\Pi^{(j)}(T)}, 0 \leq s \leq 1\right) \underset{n \rightarrow \infty}{\stackrel{d}{\longrightarrow}}\left(a_{j}^{1 / \alpha} b_{j} H_{s}^{\text {exc }}, 0 \leq s \leq 1\right),
$$

in distribution.

On the other hand, recall from the proof of Theorem 1.2 that for $n \geq 0$ and any $s \geq 0$, we have

$$
\left|H_{\lfloor \# T s\rfloor}^{T}-\frac{H_{\Lambda_{j}^{T}(\lfloor \# T s\rfloor)}^{\Pi^{(j)}(T)}}{a_{j} b_{j}}\right| \leq\left|H_{\lfloor \# T s\rfloor}^{T}-\frac{\operatorname{Anc}_{T}^{u(\lfloor \# T s\rfloor)}(j)}{a_{j} b_{j}}\right|+R_{n}(s),
$$

where

$$
\left|R_{n}(s)\right| \leq \frac{1}{a_{j} b_{j}}\left(2 \max _{0 \leq k \leq n}\left|H_{k-1}^{\Pi^{(j)}(T)}-H_{k}^{\Pi^{(j)}(T)}\right|+1\right) .
$$

Therefore, it must be clear that our claim follows from the convergence (4.6) by providing that the two terms on the right-hand side of $(4.7)$ are $o\left(n / B_{n}\right)$ in probability, uniformly in $s \in[0,1]$.

In this direction, we observe from (4.5) that $\mathbf{P}_{\text {alt }}^{(1)}\left(\# T>\delta n \mid \# T^{(j)}=n\right)=\operatorname{oe}(n)$ for any $\delta>a_{j}^{-1}$. Combining this with Proposition 2.6, we have for $0<\gamma<$ $\frac{1}{2}(1-1 / \alpha)$ and some $C>0$ that

$$
\begin{aligned}
\mathbf{P}_{\text {alt }}^{(1)} & \left(\frac{B_{n}}{n} \max _{0 \leq k \leq \# T}\left|H_{k}^{T}-\frac{\operatorname{Anc}_{T}^{u(k)}(j)}{a_{j} b_{j}}\right| \geq n^{-\frac{1}{2}(1-1 / \alpha)+\gamma} \mid \# T^{(j)}=n\right) \\
& \leq C n^{1+1 / \alpha} \mathbf{P}_{\text {alt }}^{(\mathbf{1})}\left(\frac{B_{n}}{n} \max _{0 \leq k \leq\lfloor\delta n\rfloor}\left|H_{k}^{T}-\frac{\operatorname{Anc}_{T}^{u(k)}(j)}{a_{j} b_{j}}\right| \geq n^{-\frac{1}{2}(1-1 / \alpha)+\gamma}\right)+\operatorname{oe}(n) \\
& =\operatorname{oe}(n),
\end{aligned}
$$

where $\mathbf{P}_{\text {alt }}^{(\mathbf{1})}$ is the law of alternating two-type GW forest with all its root having type 1. This shows that first term on the right-hand side of $(4.7)$ is $o\left(n / B_{n}\right)$ in probability, uniformly in $s \in[0,1]$.

Finally, let $\Upsilon^{j}$ be the number of tree components of $\Pi^{(j)}(T)$. Then the law of $\Pi^{(j)}(T)$ under the measure $\mathbf{P}_{\text {alt }}^{(1)}\left(\cdot \mid \Upsilon^{j}=r\right)$ is that of a monotype GW forest with $r$ tree components. Using Theorem 5.4 in Kortchemski and Marzouk (2016), one concludes that for $\varepsilon>0$,

$$
\lim _{n \rightarrow \infty} \mathbf{P}_{\text {alt }}^{(1)}\left(\sup _{0 \leq s \leq 1} \frac{B_{n}}{n}\left|R_{n}(s)\right| \geq \varepsilon \mid \# T^{(j)}=n, \Upsilon^{j}=r\right)=0 .
$$

By Lemma 4.4 (ii), we know that the laws of $\Upsilon^{j}$ under $\mathbf{P}_{\text {alt }}^{(1)}\left(\cdot \mid \# T^{(j)}=n\right)$ are tight as $n$ varies. Thus, we deduce that the second term on the right-hand side of (4.7) is also $o\left(n / B_{n}\right)$ in probability, uniformly in $s \in[0,1]$. 


\section{Acknowledgements}

I would like to thank Jean Bertoin for several useful discussions and for his comments on an earlier draft of this manuscript. I warmly thanks, Christina Goldschmidt and Igor Kortchemski whose suggestions and remarks helped to improve this paper. The author also thanks to the anonymous referee for his/her helpful comments.

\section{References}

R. Abraham and J.-F. Delmas. $\beta$-coalescents and stable Galton-Watson trees. ALEA Lat. Am. J. Probab. Math. Stat. 12 (1), 451-476 (2015). MR3368966.

D. Aldous. The continuum random tree. I. Ann. Probab. 19 (1), 1-28 (1991). MR1085326.

D. Aldous. The continuum random tree. III. Ann. Probab. 21 (1), 248-289 (1993). MR1207226.

K. B. Athreya and P. E. Ney. Branching processes. Dover Publications, Inc., Mineola, NY (2004). ISBN 0-486-43474-5. MR2047480.

J. Bertoin. Lévy processes, volume 121 of Cambridge Tracts in Mathematics. Cambridge University Press, Cambridge (1996). ISBN 0-521-56243-0. MR1406564.

N. H. Bingham, C. M. Goldie and J. L. Teugels. Regular variation, volume 27 of Encyclopedia of Mathematics and its Applications. Cambridge University Press, Cambridge (1989). ISBN 0-521-37943-1. MR1015093.

N. Curien and I. Kortchemski. Percolation on random triangulations and stable looptrees. Probab. Theory Related Fields 163 (1-2), 303-337 (2015). MR3405619.

R. A. Doney. On the exact asymptotic behaviour of the distribution of ladder epochs. Stochastic Process. Appl. 12 (2), 203-214 (1982). MR651904.

T. Duquesne. A limit theorem for the contour process of conditioned Galton-Watson trees. Ann. Probab. 31 (2), 996-1027 (2003). MR1964956.

T. Duquesne and J.-F. Le Gall. Random trees, Lévy processes and spatial branching processes. Astérisque (281), vi+147 (2002). MR1954248.

W. Feller. An introduction to probability theory and its applications. Vol. II. Second edition. John Wiley \& Sons, Inc., New York-London-Sydney (1971). MR0270403.

P. Jagers. General branching processes as Markov fields. Stochastic Process. Appl. 32 (2), 183-212 (1989). MR1014449.

S. Janson and S. Ö. Stefánsson. Scaling limits of random planar maps with a unique large face. Ann. Probab. 43 (3), 1045-1081 (2015). MR3342658.

I. Kortchemski. Invariance principles for Galton-Watson trees conditioned on the number of leaves. Stochastic Process. Appl. 122 (9), 3126-3172 (2012). MR2946438.

I. Kortchemski. A simple proof of Duquesne's theorem on contour processes of conditioned Galton-Watson trees. In Séminaire de Probabilités XLV, volume 2078 of Lecture Notes in Math., pages 537-558. Springer, Cham (2013). MR3185928.

I. Kortchemski. Sub-exponential tail bounds for conditioned stable BienayméGalton-Watson trees. Probab. Theory Related Fields 168 (1-2), 1-40 (2017). MR3651047.

I. Kortchemski and C. Marzouk. Triangulating stable laminations. Electron. J. Probab. 21, Paper No. 11, 31 (2016). MR3485353. 
I. Kortchemski and C. Marzouk. Simply generated non-crossing partitions. Combin. Probab. Comput. 26 (4), 560-592 (2017). MR3656342.

J.-F. Le Gall. Random trees and applications. Probab. Surv. 2, 245-311 (2005). MR2203728.

J.-F. Le Gall and G. Miermont. Scaling limits of random planar maps with large faces. Ann. Probab. 39 (1), 1-69 (2011). MR2778796.

G. Miermont. Invariance principles for spatial multitype Galton-Watson trees. Ann. Inst. Henri Poincaré Probab. Stat. 44 (6), 1128-1161 (2008). MR2469338.

J. Pitman. Combinatorial stochastic processes, volume 1875 of Lecture Notes in Mathematics. Springer-Verlag, Berlin (2006). ISBN 978-3-540-30990-1; 3-54030990-X. MR2245368.

L. de Raphélis. Scaling limit of multitype Galton-Watson trees with infinitely many types. Ann. Inst. Henri Poincaré Probab. Stat. 53 (1), 200-225 (2017). MR3606739.

G. Samorodnitsky and M. S. Taqqu. Stable non-Gaussian random processes. Stochastic Modeling. Chapman \& Hall, New York (1994). ISBN 0-412-05171-0. MR1280932.

K. Sato. Lévy processes and infinitely divisible distributions, volume 68 of Cambridge Studies in Advanced Mathematics. Cambridge University Press, Cambridge (2013). ISBN 978-1-107-65649-9. MR3185174. 